\title{
Continuous Aerodynamic Modelling of Entry Shapes
}

\author{
Dominic Dirkx* and Erwin Mooij ${ }^{\dagger}$ \\ Delft University of Technology, Delft, 2600 AA, The Netherlands
}

\begin{abstract}
During the conceptual design phase of a re-entry vehicle, the vehicle shape can be varied and its impact on performance evaluated. To this end, the continuous modeling of the aerodynamic characteristics as a function of the shape is useful in exploring the full design space. Local inclination methods for aerodynamic analysis have proven sufficiently accurate for use at such a design stage, but manual selection of methods over the vehicle is inefficient for the exploration of a large number of design possibilities. This paper describes the model of an aerodynamic analysis code, written for use in conceptual vehicle shape optimization, which includes an automatic method selection algorithm. Panel shielding is also included in the analysis code to allow for the analysis of more complex geometries. The models used for the shape and aerodynamics are described and results for the Space Shuttle and Apollo are compared to wind tunnel data. They show an accuracy of better than $15 \%$ for most cases, which is sufficient for the use in conceptual design. Panel shielding is shown to be important in the prediction of control derivatives at low angle of attack, as well as the prediction of lateral stability derivatives. Finally, a simple guidance algorithm is used to assess the impact of the errors in the aerodynamic coefficients on the vehicle heat load and ground track length. Both show discrepancies of less than $10 \%$.
\end{abstract}

\section{Nomenclature}

$\begin{array}{ll}A & =\text { Area }\left[\mathrm{m}^{2}\right] \\ C_{p} & =\text { Pressure coefficient }[-] \\ C_{D} & =\text { Drag coefficient }[-] \\ C_{L} & =\text { Lift coefficient }[-] \\ C_{m} & =\text { Pitch moment coefficient }[-] \\ c_{r e f} & =\text { Doment reference length }[\mathrm{m}] \\ D & =\text { Cubic bridging polynomial }[-] \\ f_{c}(t) & =\text { Acceleration due to gravity }[\mathrm{m}] \\ g & =\text { Altitude }[\mathrm{m}] \\ h & =\text { method selection parameter }(i=1 . .9)[-] \\ k_{i} & =\text { Length }[\mathrm{m}] \\ L & =\text { Mach number }[-] \\ M & =\text { Mass }[\mathrm{kg}] \\ m & =\text { Convective heat rate }\left[\mathrm{W} / \mathrm{m}^{2}\right] \\ q_{c} & =\text { Radius }[\mathrm{m}] \\ R & =\text { Distance to moment reference point }[\mathrm{m}] \\ \mathbf{r} & =\text { Tement reference area }\left[\mathrm{m}^{2}\right] \\ S_{r e f} & =\text { Velocity [m/s] } \\ T & \\ \mathbf{V} & { }^{*} \text { Graduate Student. Currently: Researcher, Faculty of Aerospace Engineering, d.dirkx@tudelft.nl } \\ \dagger \text { Assistant Professor, Faculty of Aerospace Engineering, e.mooij@tudelft.nl, Senior member AIAA. }\end{array}$




$\begin{array}{lll}\alpha & =\text { Angle of attack [rad] } \\ \beta & =\text { Sideslip angle [rad] } \\ \beta & =\text { Shock angle [rad] } \\ \delta_{b f} & =\text { Body flap deflection [rad] } \\ \delta_{e} & =\text { Rlevon deflection [rad] } \\ \gamma & =\text { Flight-path angle [rad] } \\ \gamma & =\text { Latitude [rad] } \\ \delta & =\text { Lateral panel inclination }[\mathrm{rad}] \\ \epsilon & =\text { Panel inclination angle }[\mathrm{rad}] \\ \theta & =\text { Cone half angle [rad] } \\ \theta_{c} & =\text { Wing sweep angle [rad] } \\ \Lambda & \left.=\text { Density [kg/m }{ }^{3}\right] \\ \rho & =\text { Bank angle }[\mathrm{rad}] \\ \sigma & =\text { Longitude }[\mathrm{rad}] \\ \tau & =\text { Heading angle }[\mathrm{rad}] \\ \chi & =\text { Central body angular rate }[\mathrm{rad} / \mathrm{s}] \\ \omega_{P} & \end{array}$

\section{Subscripts:}

$\begin{array}{ll}\operatorname{cog} & =\text { Center of gravity } \\ R & =\text { In inertial Earth-fixed frame } \\ s & =\text { At stagnation point }\end{array}$

\section{Introduction}

During the conceptual design stage of re-entry vehicles, the design parameters are varied to gain knowledge of their effect on the vehicle's overall performance and its capability to fulfill the design requirements. One of the defining characteristics of an entry vehicle is its shape, as this shape will largely define the aerothermodynamic characteristics of the vehicle. Since aerothermodynamic challenges, such as vehicle heating remain one of the most difficult problems in atmospheric re-entry, an exploration of the possible shapes for a vehicle early in the design is advisable. It is advantageous to use a continuous model for the analysis, so that one is not limited to the analysis and comparison of a limited number of shapes, ${ }^{1}$ but is instead free to analyze any shape in the design space. This paper will discuss a simplified aerodynamic model, which is to be used in a vehicle-optimization effort. The optimization is to be carried out on both a capsule-shaped vehicle described in this paper, as well as a freeform winged fuselage entry vehicle using spline surfaces. ${ }^{2}$

The validation of the aerodynamics code through comparison with Apollo and Space-Shuttle wind-tunnel data will be used here to assess the accuracy of the methodology that is used. The effect that the differences will have on vehicle trajectories will also be adressed, to assess the capability of the aerodynamic models to properly predict the vehicles' performance criteria in an optimization loop. The definition of the shapes is discussed in Section II, followed by the definition of the aerothermodynamic models in Section III. Section IV will show the comparison between the calculated and measured aerodynamic coefficients of Apollo and the Shuttle, and give a discussion of the discrepancies that are observed. The trajectory and guidance models are described in Section V, whereas Section VI will show the impact of the aerothermodynamic discrepancies on the resulting trajectories. Finally, Section VII will conclude the paper with conclusions and recommendations for future work. 


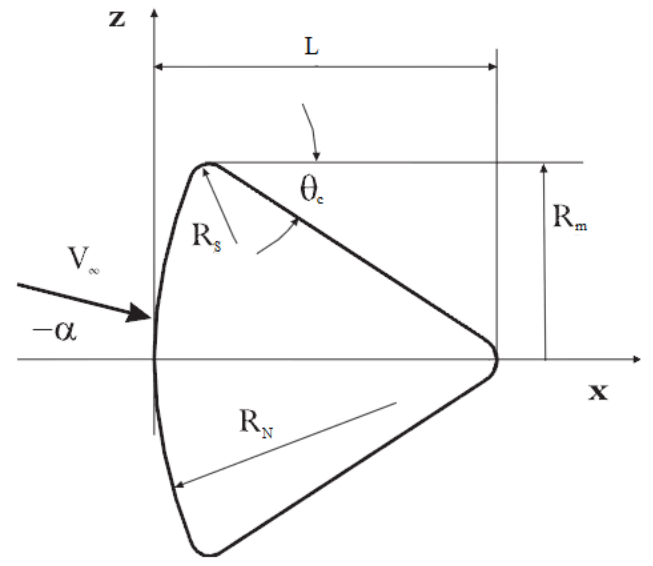

(a)

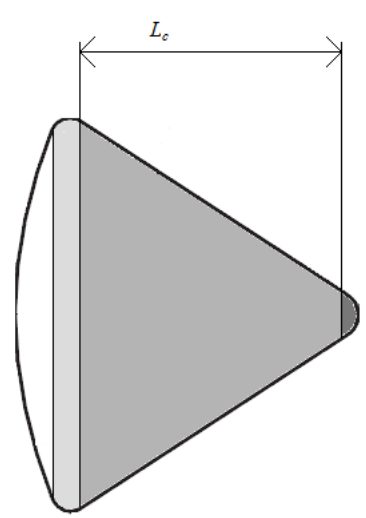

(b)

Figure 1. Schematic representation of capsule re-entry vehicle shape ${ }^{4}$ a) Vehicle parameters b) Division in analytical shapes

\section{Geometry Definition}

The definition and parametrization of the vehicle shape is the starting point of the analysis of the problem. Numerous possibilities exist for defining vehicle shapes, where in this context the continuous variation of a limited number of parameters on which it is to be based is crucial. In the optimization, two cases will be treated, one describing analytical shape definitions and one using freeform spline definitions. The former of these will be used for the validation of the aerodynamics model in this article, while the latter will be considered in future work. For validation of the code for a lifting entry vehicle, a Space-Shuttle mesh will be used, which is independent of the shape parametrization that will be used in the optimization.

\section{II.A. Capsule Shape}

A number of vehicle shapes can be defined by a combination of analytical surfaces. An example of this in a framework similar to the one discussed here is found in literature. ${ }^{3}$ The analytical shape that will be used in this study is shown in Fig. 1. This is a parametrization of, among others, the Apollo capsule. As can be seen, it consists of four matched analytical geometries, a sphere segment, a torus segment, a conical frustum and a spherical segment. Although no unique set of parameters exists for defining this shape, the required number of parameters for defining it is five. Since the shape is axisymmetric, the full surface geometry is defined by the cross-section shown. The parameters which are chosen for the shape definition are:

- Nose radius $R_{N}$

- Side radius $R_{S}$

- Rear cone half-angle $\theta_{c}$

- Mid radius $R_{m}$

- Rear conical part length $L_{c}$

The parameter $L_{c}$ is chosen here, instead of the capsule length $L$, due to the simplicity of the constraint on it from other vehicle parameters. This will be advantageous in the optimization process. The cross-sections of the constituent analytical shapes can be seen from Fig. 1(b). The relations between the parameters of the capsule and the parameters of the analytical shapes can be derived by simple geometrical relations.

When generating a capsule shape, the constraints on the parameters are inter-dependent. That is, in addition to constraints imposed on each variable a priori, the choice of $R_{N}$ and $\theta_{c}$ influence the available choices of $R_{m}$ and $L_{c}$. When generating the variables in the order described above, the following constraints 

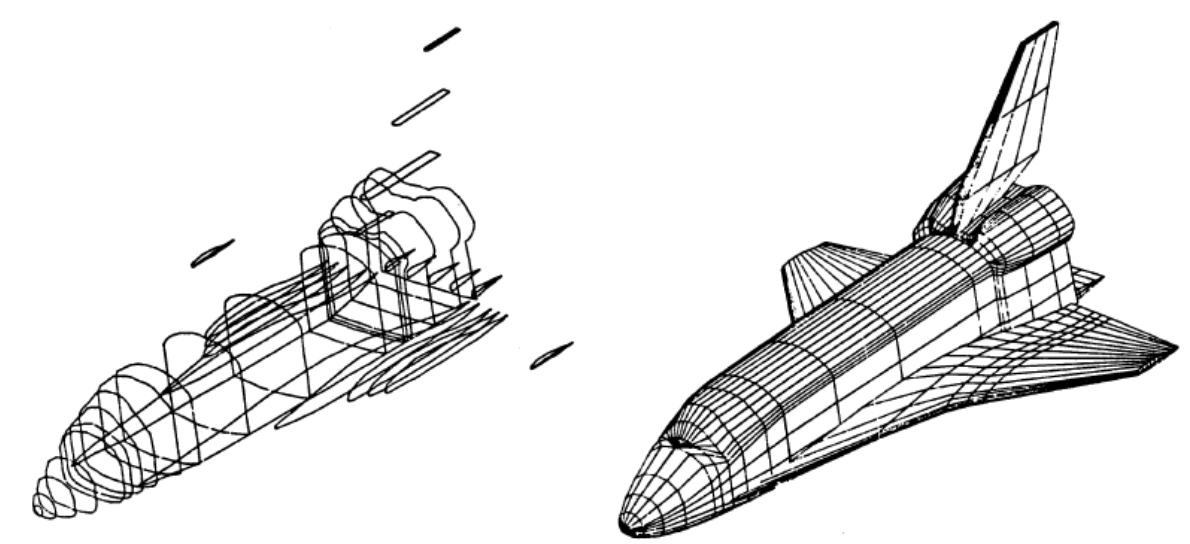

Figure 2. Space Shuttle wireframe model from Langley Wireframe Geometry Standard, note the difference in the formation of fusiform and planar geometries. ${ }^{5}$

are to be observed:

$$
\begin{aligned}
R_{m} & <R_{N} \\
L_{c} & <\frac{R_{m}-R_{S}\left(1-\cos \theta_{c}\right)}{\tan \theta_{c}}
\end{aligned}
$$

In addition to the external geometry of the vehicle, the capsule's center of mass is important for determining the vehicle's performance. Its position is parametrized by two variables, i.e., its position along the vehicle centerline and the offset from this centerline.

\section{II.B. Surface Mesh}

For the aerodynamic analysis described in the next section, a panelled surface mesh is required. The geometry input file format that is used is the Langley Wireframe Geometry Format (LaWGS). ${ }^{5}$ It bases the definition of a wireframe on a discrete number of points which make up an object. The entire configuration can then be defined from an arbitrary number of these objects. Objects may be defined in a (right-handed Cartesian) global coordinate system, or a local coordinate system, where the specification of the translation and rotation from the local to global coordinate system must be given. In addition, a mirror symmetry about the $x y$-, $x z$ - or $y z$-plane may be defined in either local or global coordinates for an object.

A single object is composed of a number of contours, which are in turn composed of a number of points, where the number of points on each contour must be equal. By connecting each point to both neighbouring points on the same contour and connecting points with the same indices on subsequent contours, a wireframe of quadrilaterals is obtained. An example of a resulting wireframe for the Space Shuttle is shown in Fig. 2.

Although not required for the file format, a guideline, which is later exploited in the aerodynamics code (see Section III.B), is to define parts as either 'fusiform' or 'planar'. The former includes fuselages, etc., and the latter includes wings, vertical and horizontal stabilizers, etc. The distinction between these two types of geometries can be seen in Fig. 2, where the different orientations of the contours on the wings, flap and tail versus the fuselage are clearly indicated.

\section{Aerothermodynamics}

The aerothermodynamic effect on a re-entry vehicle in hypersonic flow is characterized by the aerodynamic forces and moments, as well as the heating over the vehicle. For use in trajectory simulation, a database of coefficients as a function of freestream Mach number $M$, angle of attack $\alpha$ and sideslip angle $\beta$ is generated, and these values are interpolated to yield a data point at a particular flight condition during integration.

For a complete aerothermodynamic analysis, the prediction of pressure, friction and heating on the surface should be performed in unison, as these effects are strongly coupled in hypersonic flow. To perform such an analysis would require the use of a Computational Fluid Dynamics (CFD) tool, which numerically solves 
the (chemically reacting) Navier Stokes equations. Such an analysis would require a prohibitive amount of time in the context of the vehicle optimization work, however, since aerodynamic coefficients are required for all attitudes and Mach numbers that occur during the re-entry of each of the vehicle configurations to be analyzed. Depending on the exact number of trajectory integrations during the optimization, as well as the total number of Mach numbers and attitude angles at which coefficients are determined, aerodynamic coefficients for $10^{5}-10^{7}$ cases will need to be computed. Obviously, this is infeasible in a reasonable amount of time using a Navier-Stokes solver, or even an Euler solver.

For this reason, a set of simple analysis methods for hypersonic flow are used in this study, notably local inclination methods. The rationale behind these methods, as well as their implementation, will be described in Section III.A. An algorithm for the selection of the applicable aerodynamic method per vehicle part is discussed in Section III.B. A method that has been used in the past as a modification of the local inclination methods is discussed in Section III.C. Finally, the heating analysis, which in this study is limited to stagnation-point heating, is given in Section III.D.

A first version of software was developed in the framework of the open source software project Space Trajectory Analysis (STA) ${ }^{6}$ as the Re-entry Aerodynamics Module (RAM). This version includes the method selection algorithm, but does not include the panel shielding or the ability to determine control derivatives.

The local inclination methods produce only a pressure distribution on the vehicle. The viscous effect on the aerodynamic forces and moments will be neglected in this study. Additionally, the flow will be assumed to be a continuum throughout the entry. The methods described in the next section will be used to calculate pressure coefficients $C_{p}$ on each of the vehicle panels.

\section{III.A. Local Inclination Methods}

An important set of force estimation methods in hypersonic flow is formed by the so-called local inclination methods, which require only the angle $\theta$ by which the surface is (locally) inclined w.r.t. the free stream flow to produce a pressure coefficient. Although such methods are obviously highly simplified, reasonable results can be obtained by using them. Due to this combination of simplicity and reasonable validity, this type of method finds wide use in conceptual design ${ }^{7-11}$ and are very well suited to use in conceptual design optimization. The long heritage has also made them well documented. An excellent introduction is given in literature, ${ }^{12}$ with their formulation and implementation extensively discussed in manuals for previous codes using such methods. ${ }^{13-15}$ Generally, two classes of methods are used, one for the windward side and one for the leeward side. The leeward side methods are also applied to 'shielded' sections. Shielding occurs when a vehicle is oriented to the flow in such a manner that a part of it is 'shielded' from the oncoming flow by another part of it. In such cases, the windward surface-inclination method should be applied only to the surface the flow encounters first, the second part should use an appropriate leeward method.

The most basic inclination method for the estimation of the aerodynamic forces and moments on a hypersonic body is the Newtonian method. This method assumes that, upon hitting a surface, the flow

loses its component normal to the surface while retaining all of its tangential motion. The assumptions of Newtonian flow lead to the following relation for the local pressure coefficient:

$$
C_{p}=2 \sin ^{2} \theta
$$

A modification of this method that is typically used involves an additional physical consideration of supersonic flow, namely the loss of total pressure over a shock wave. Including the loss of total pressure will increase the physical justification of the model. The model, which is obtained is termed the Modified Newtonian method, the pressure coefficient it produces is:

$$
C_{p}=C_{p, s} \sin ^{2} \theta
$$

where $C_{p, s}$ is the stagnation point pressure coefficient.

The modified Newtonian method assumes that that all streamlines have passed through a normal shock wave. Although this assumption will in general be true for only a very select number of streamlines, Fig. 3 (a) gives an indication as to why this assumption can be assumed valid as a first-order approximation over a larger region. Here, the stagnation pressure coefficient that occurs after passing through an oblique shock wave is plotted. It can be seen that the 'correct' value of $C_{p, s}$ varies very little over the range $40^{\circ}<\theta<90^{\circ}$. For low shock angles (and therefore low inclination angles), however, the value of $C_{p, s}$ increases very quickly. 


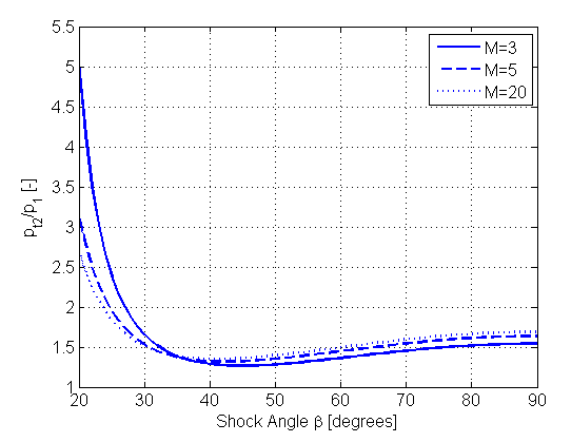

(a)

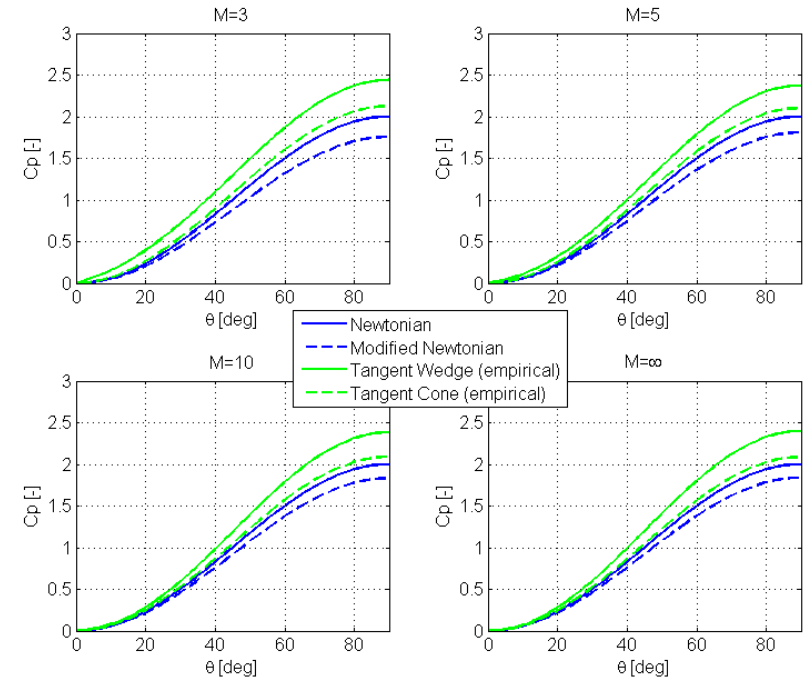

(b)

Figure 3. a) Ratio of post-shock total pressure to freestream pressure, $\gamma=1.4$ b) Effect of local inclination method on pressure prediction, $\gamma=1.4$

For the cases of low inclination angles, where the flow has typically (but not necessarily) passed through a low-angle shock wave, different local inclination methods are typically used. The two most popular methods, the Tangent Wedge and Tangent Cone methods, ${ }^{13}$ liken the local flow to that of an equivalent wedge or cone. Although the cone and wedge will (for sufficiently low cone and wedge angles) have an attached nose shock, these methods have also seen use in predicting the pressure on the rear part of a blunt nosed shape, which will have a detached shock. This is due to the fact that for such a case, as for instance a vehicle fuselage, the shock angle of the shock for which the flow impacting a surface at these low inclination region has passed through will be similar to that which it would have passed through had there been an attached shock. Even though the flowfield behind the shock will be different in the two cases and the development of the boundary and entropy layer will differ, using these methods can still produce results which are superior to those produced by the (Modified) Newtonian method, and are therefore useful in a conceptual design stage. Pressure coefficients for the three methods discussed are shown in Fig. 3(b). It can be seen that the general trend with inclination angle is similar for all methods, but the scale of the results differ.

For the determination of the expansion pressure coefficient of the vehicle, a different method must be used. A lower bound for the pressure coefficient is taken as the vacuum pressure coefficient:

$$
C_{p, v a c}=-\frac{2}{\gamma M_{\infty}^{2}}
$$

However, since there will be some flow on the rear of the vehicle due to, for instance, flow recirculation, this will underpredict the occuring pressure. An empirical correlation, which is sometimes used, is:

$$
C_{p}=-\frac{1}{M_{\infty}}
$$

Another method that can be used is Prandtl-Meyer expansion from the point of $\theta=0$ onwards. This method is slightly more complex than the above methods, because it is also a function of the inclination angle $\theta$.

In general, different methods are applicable for low and high hypersonic Mach numbers, the details of this will be described in Section III.B. To have a database of aerodynamic coefficients that is continuous in $M$, a bridging between the low and high hypersonic aerodynamic coefficients is employed, in a similar fashion to what is sometimes employed for the transitional region between rarefied and continuum flow. A cubic bridging polynomial $f_{c}(t)$, with $t$ running from 0 to 1 , is used. To match both the values and the slopes of the coefficients at the boundaries of the bridging domain, $f_{c}(t)$ is chosen to be:

$$
f_{c}(t)=-2 t^{3}+3 t^{2}
$$


Table 2. Selection of applicable methods per vehicle part type

\begin{tabular}{ccc}
\hline \hline & Low Hypersonic Compression & High Hypersonic Compression \\
\hline Blunt & Modified Newtonian & Modified Newtonian \\
Low inclination 'round' & Tangent Cone & Modified Newtonian \\
Low inclination 'flat' & Tangent Wedge & Modified Newtonian \\
\hline \hline & Low Hypersonic Expansion & High Hypersonic Expansion \\
\hline Blunt & ACM Empirical & High Mach Base pressure \\
Low inclination 'round' & Prandtl-Meyer expansion & Prandtl-Meyer expansion \\
Low inclination 'flat' & Prandtl-Meyer expansion & Prandtl-Meyer expansion \\
\hline \hline
\end{tabular}

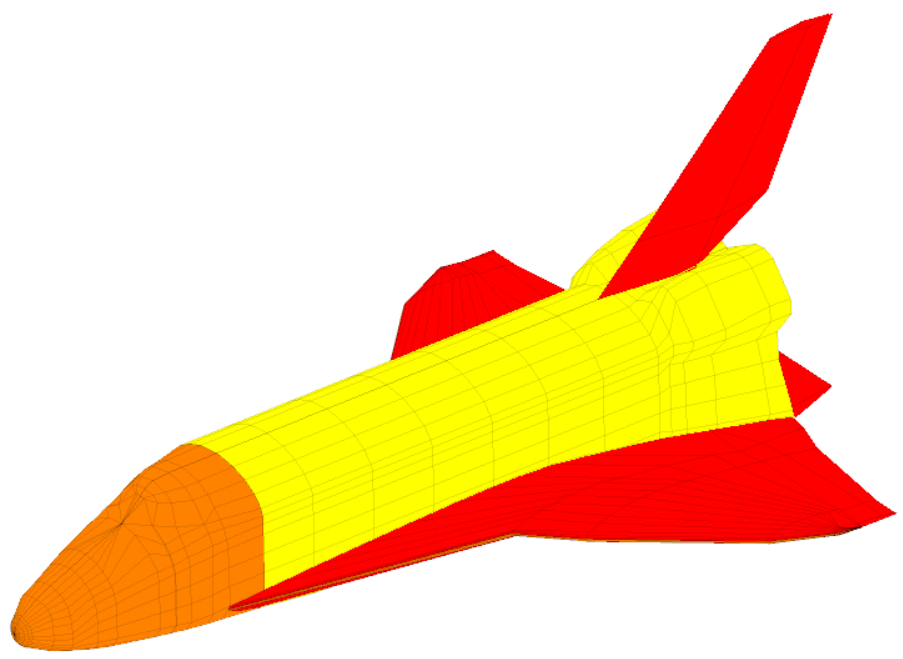

Figure 4. Geometry type identification on Space Shuttle configuration. Dark to light (red-orange-yellow) low inclination flat, blunt, low inclination curved.

To use this method, it is required that there is an overlap between the low and high hypersonic regimes. In the overlap region $M_{h i g h, \min }$ to $M_{l o w, \max }$, the following is used when an aerodynamic coefficient $C_{i}$ is retrieved:

$$
\begin{aligned}
& t=\frac{M-M_{\text {low, } \max }}{M_{\text {high }, \min }-M_{\text {low } \max }} \\
& C_{i}=C_{i, \text { high }} f_{c}(t)+\left(1-f_{c}(t)\right) C_{i, \text { low }}
\end{aligned}
$$

\section{III.B. Method Selection}

Although the use of local inclination methods has been common in conceptual hypersonic aerodynamics, there is still no clear quantified consensus on when exactly to use which method. Due to the variation in the results produced by the various methods, it is important to make a good determination of which method to use on which vehicle part. An automatic selection algorithm has been developed, which analyzes the vehicle shape and assigns regions where each of the various methods is to be applied. After the guidelines found in, literature, ${ }^{16-18}$ as well as a number of tests for, among others, the geometries described here, the applicable methods are chosen to be as in Table 2. A description of the algorithm is given in Appendix A.

The algorithm has been used successfully to determine the choice of local inclination methods according to Table 2, similarly to how a human would apply these methods. An example of selected methods is shown in Fig. 4 


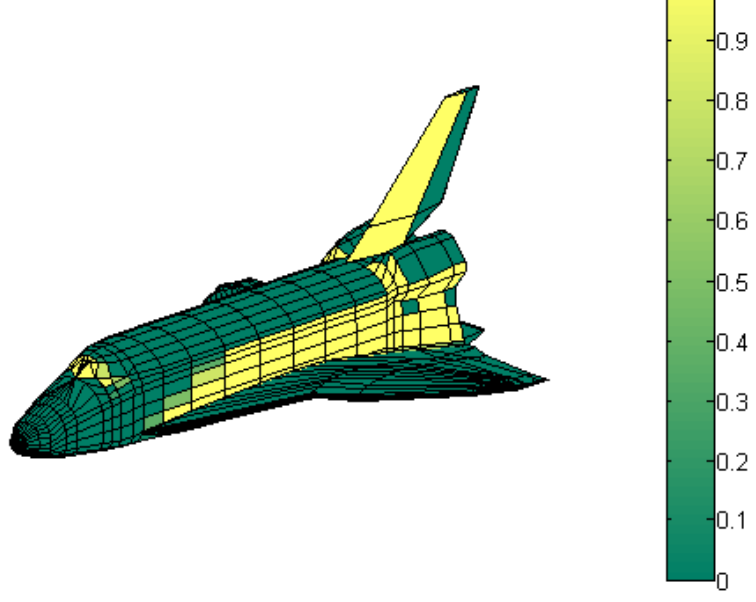

(a)

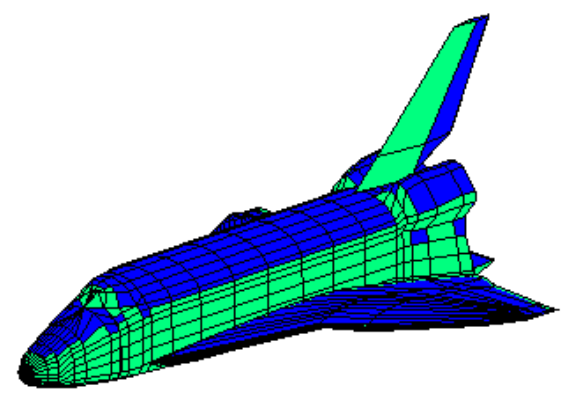

(b)

Figure 5. Shadowing of Space Shuttle panels, $\alpha=30^{\circ}, \beta=2^{\circ}$, a) Shadowed fraction per panel b) Panel type, green $=$ compression, blue = expansion

\section{III.C. Panel Shielding}

For complex geometries with protrusions such as wings, vertical stabilizer(s) or a body flap, the concept of panel shielding can become important in determining the aerodynamic coefficients. For the standard local inclination methods, any panel with a positive inclination angle is treated as a compression panel. When two panels are (partially) behind one another, though, this assumption needs to be revised. When a given freestream streamline is extended through the vehicle geometry and intersects multiple panels with positive inclination, it is obvious that it will only have the true compression effect on one of these panels. This can be clarified by considering the Newtonian method, which is based on the loss of momentum normal to the wall and realizing that a given flow volume can only lose this momentum once.

To account for this effect an algorithm has been used, ${ }^{13}$ by which all combinations of panels are analyzed and the occurrence and size of overlapping regions are determined. The algorithm is capable of quickly discarding the possibility of shielding when comparing two panels to increase computational efficiency. The procedure described there proved to be insufficient for the comparison of two panels where vertices of the two panels that are being compared coincide or if a vertex of one panel is on the side of the other. Criteria to account for these cases have been implemented and tested in the analysis code. The exact procedure will not be explained in detail here for the sake of brevity.

As can be imagined, the computational effort required for the shielding analysis can be significant, as it requires each combination of panels to be analyzed, so that the computational time will increase with $n^{2}$, whereas determining the pressures on the panels using the local inclination methods only takes computational time on the order of $n$. For this reason, it should only be used when it is expected to have an important influence on the results in question.

Due to the limited usefulness and the high computational cost, the calculation of multiple shielding, where one panel is shielded by more than one panel, is not considered here. Although this will cause some errors in the results, the occurrence of such a case has proven to be rare, so that the influence is negligible. An example of the panel-shadowing determination is shown in Fig. 5, where the shadowed fraction of each Shuttle panel is shown, along with a figure showing which panel is a compression surface, and which is an expansion surface.

\section{III.D. Heating Analysis}

The aerodynamic heating of entry vehicles poses a serious design challenge due to the high heat fluxes that are reached during re-entry. For a detailed analysis of hypersonic heating over a full configuration, CFD or 

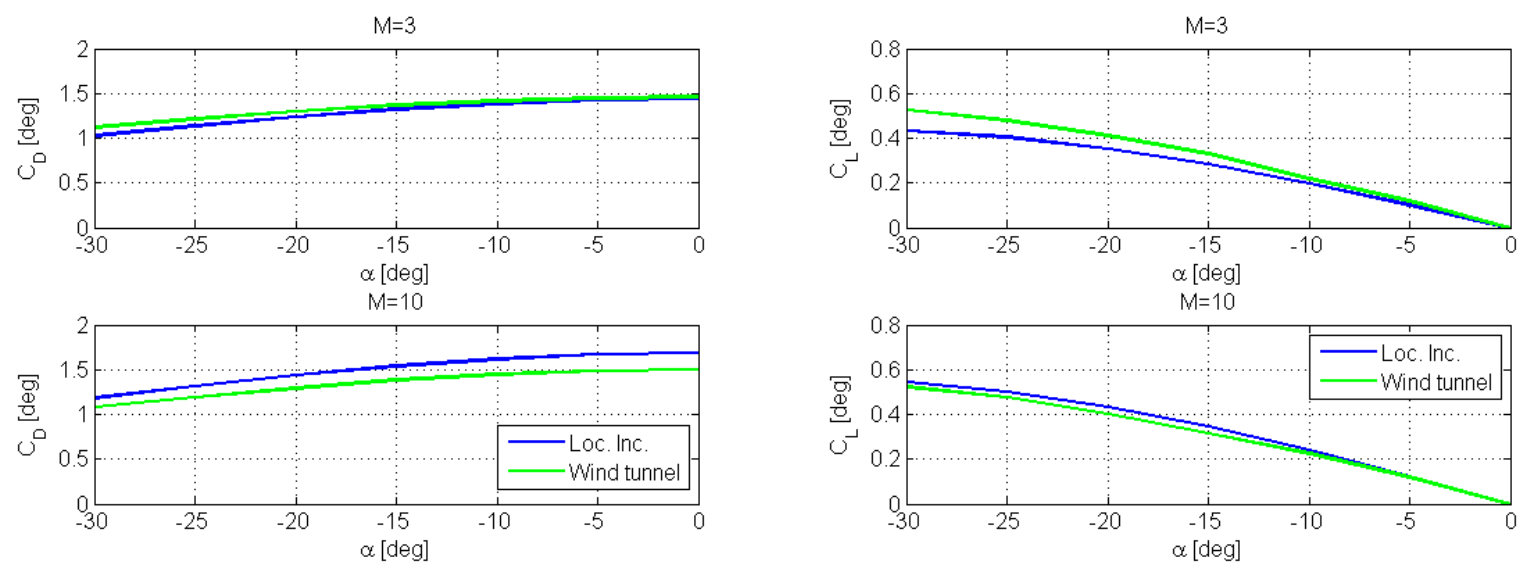

(a)

(b)
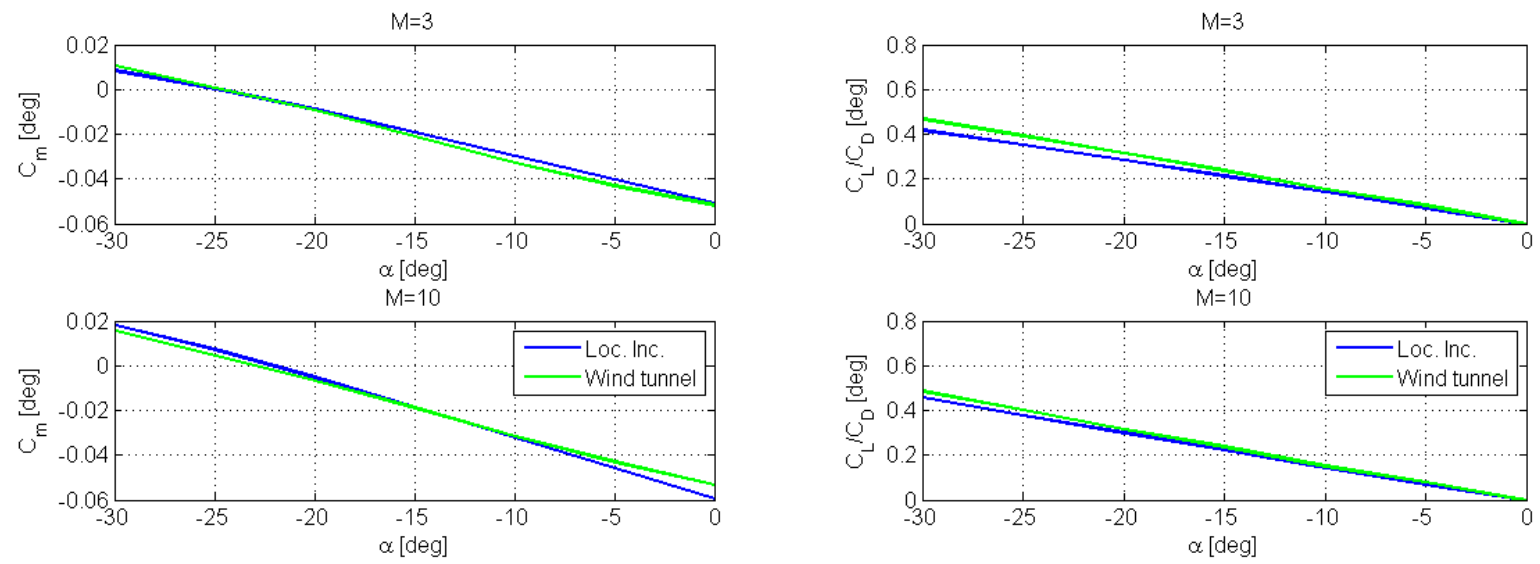

(c)

(d)

Figure 6. Aerodynamic coefficients for Apollo command capsule a) Drag coefficient b) Lift coefficient c) Moment coefficients d)Lift over Drag

experimental data are required, which is not feasible for this project. Instead, a number of (semi-)empirical correlations, which have been extensively used in conceptual vehicle design, will be employed here. The following relation is used for convective heating at a stagnation point:

$$
q_{c, s}=k \rho^{N_{1}} V^{N_{2}}
$$

where the values of $k, N_{1}$ and $N_{2}$ that are used in literature vary somewhat. The values used here, assuming laminar flow conditions, are $N_{1}=0.5$ and $N_{2}=3 .{ }^{19}$ For $k$ the following relation is used:

$$
k=\frac{1.83 \cdot 10^{-4}}{\sqrt{R_{n}}}\left(1-\frac{T_{w}}{T_{a w}}\right)
$$

where $R_{n}$ is the nose radius, $T_{w}$ the wall temperature and $T_{a w}$ the adiabatic wall temperature. As a first approximation, the cold wall approximation can be used, so that $T_{w} / T_{a w} \approx 0$ is assumed.

\section{Aerodynamic Verification and Validation}

The aerodynamic coefficients produced by the methods described in Section III.B are compared to available aerodynamic databases and the influence on the discrepancies is analyzed. 


\section{IV.A. Apollo}

The geometry of the Apollo capsule has been modelled according to the parametrization given in Section II.A, with the parameters taken from literature ${ }^{4}$ to be the following:

$R_{N}=4.694 \mathrm{~m}, R_{m}=1.956 \mathrm{~m}, R_{S}=0.196 \mathrm{~m}, \theta_{c}=33^{\circ}, L_{c}=2.662 \mathrm{~m}$

Aerodynamic coefficients were then generated and compared to the data from wind-tunnel testing. ${ }^{20}$ The reference quantities used in this reference, as well as here are:

$S_{\text {ref }}=39.441 \mathrm{~m}^{2}, c_{\text {ref }}=3.9116 \mathrm{~m}, \mathbf{r}_{\mathbf{c o g}}=(1.0367,0,0.1369) \mathrm{m}$

where the axes are defined as in Fig. 1.

Figs. 6(a) - 6(b) show the longitudinal translational coefficients of the Apollo capsule compared to the wind-tunnel results. It can be seen that the results coincide reasonably well with the wind tunnel data. The most important difference between the two is the over-prediction at $M=10$. At this Mach number the contribution of the expansion is minimal. This is due to the fact that the vacuum pressure coefficient is very close to zero and the expansion pressure coefficient will therefore not differ from 0 by much, so that the results are almost fully defined by the compression pressure distribution. From Fig. 3(b), it can be seen that the Modified Newtonian method gives the lowest value of the pressure coefficient at a given inclination angle of the methods used. Since this method is used on all compression surfaces at $M=10$ and still produces an axial force which is too high, this indicates that no suitable local inclination method exists which can produce sufficiently low results here. This could be the result of the fact that stream-lines that pass through a shock wave of inclination angle somewhat lower than $90^{\circ}$ (which will be the case for the detached bow shock) results in a lower stagnation pressure coefficient. This will in turn result in a lower pressure coefficient. This effect has not been included in the code, but could be part of future work. The effect of this can be seen to be acceptable, though. Although not included in this paper, it was observed that the aerodynamic coefficients for the EXPERT ${ }^{21}$ capsule differed by a greater amount, most likely due to a related phenomenon.

The moment coefficients for the Apollo capsule are shown in Fig. 6(c). It can be seen that the results coincide very well with the wind tunnel data. In this study, since attitude propagation is not included and only vehicle trim is considered to determine the attitude, it is important that the trimmed $\alpha$ and consequent $L / D$ are well predicted. From the figure, it can be seen that the value of $\alpha_{\text {trim }}$ is off by about $1^{\circ}$ for $M_{\infty}=10$ and $0.1^{\circ}$ for $M_{\infty}=3$. The resulting errors in $L / D$ are $\sim 10 \%$ in both cases. These discrepancies are more than acceptable for a conceptual design effort, and their influence on the trajectories of the capsule will be discussed in Section VI.
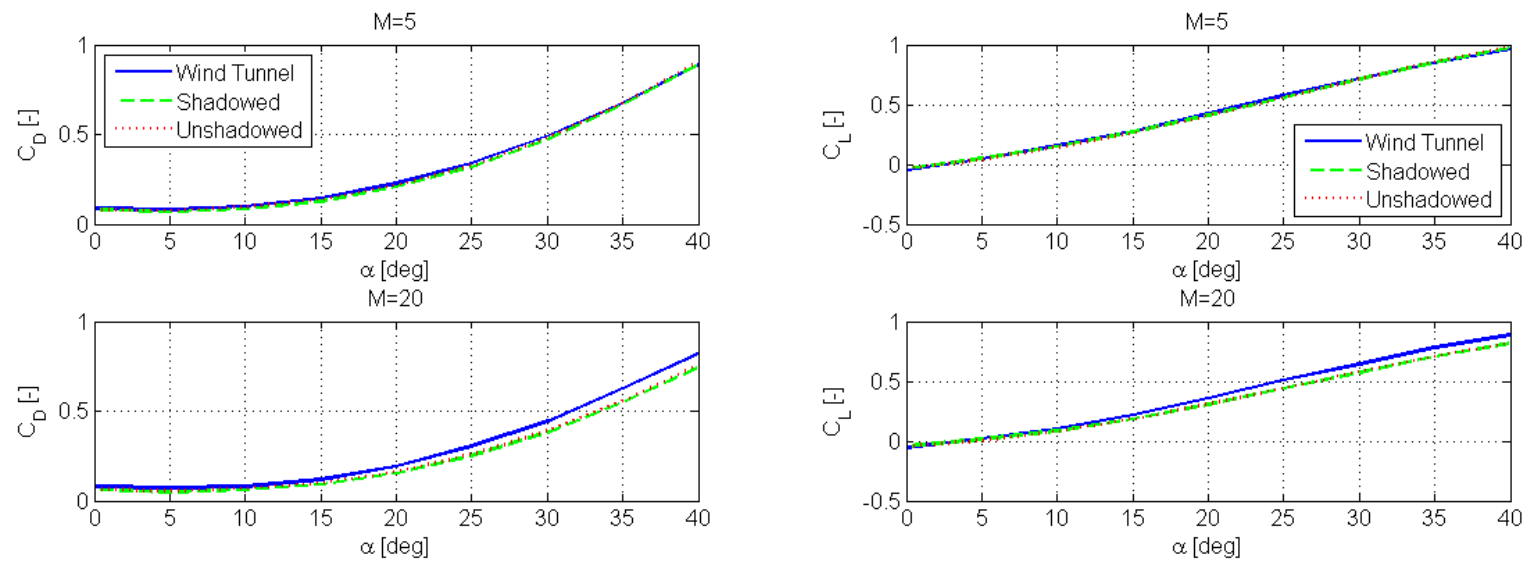

(a)

(b)

Figure 7. Lift and drag coefficients produced for the Space Shuttle with and without shadowing compared to wind tunnel data 

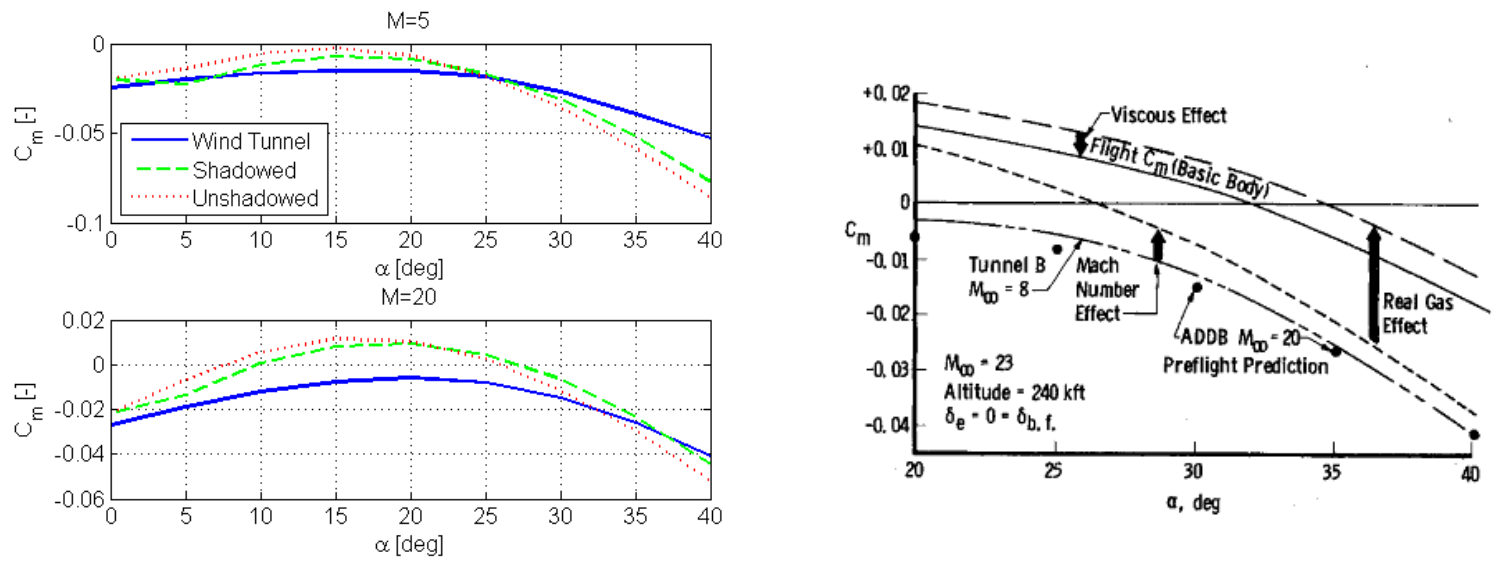

(a)

(b)

Figure 8. Moment coefficients for the Space Shuttle a) Results generated here with and without shadowing compared to wind tunnel data b) Wind tunnel data compared to flight data ${ }^{22}$
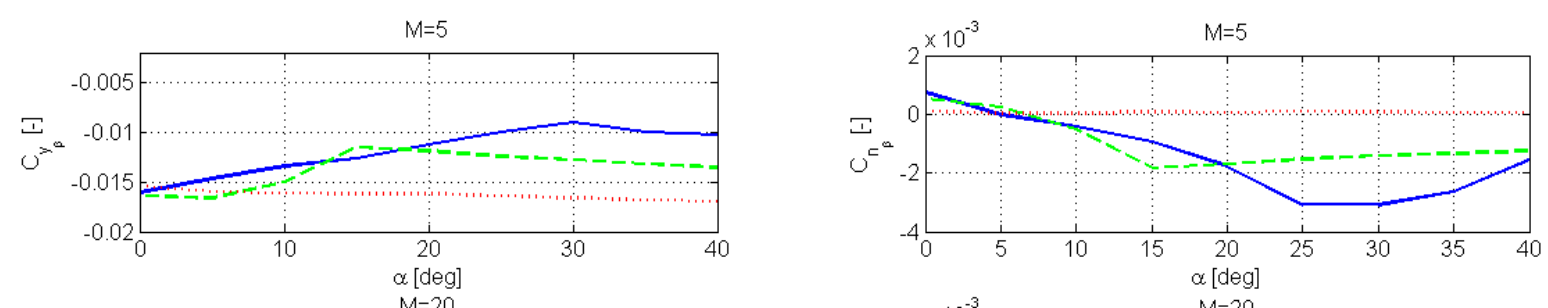

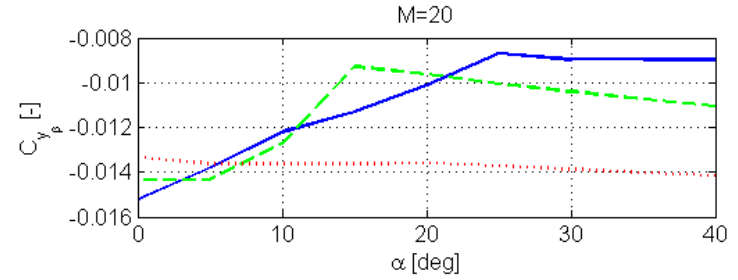

(a)

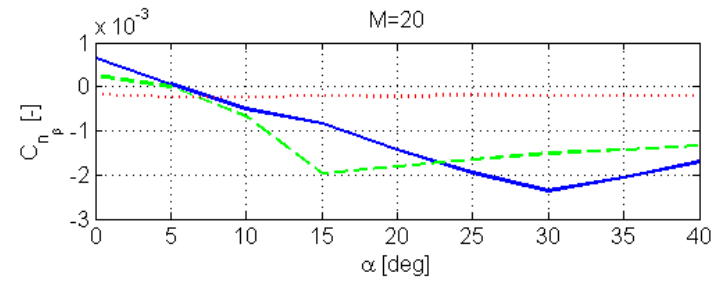

(b)
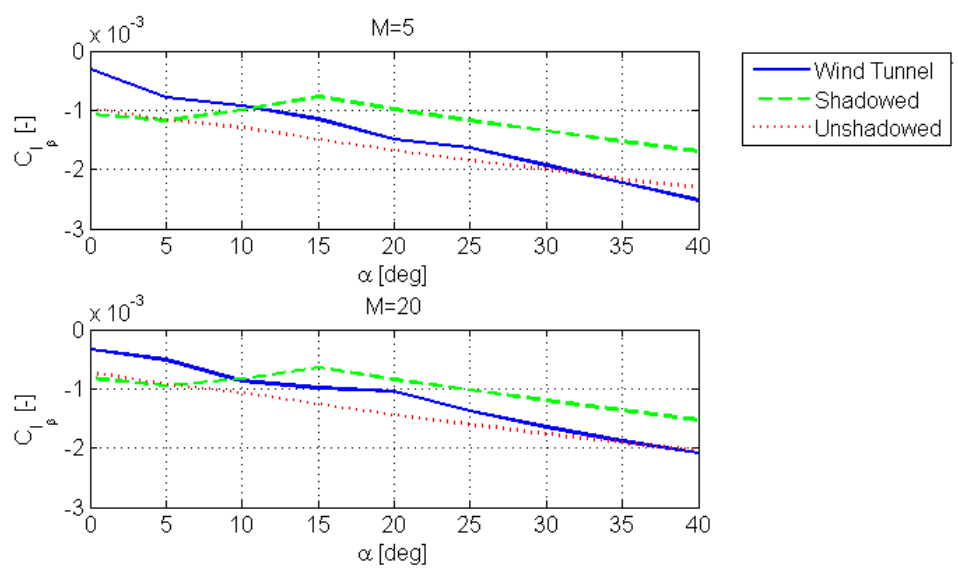

(c)

Figure 9. Stability derivatives produced for the Space Shuttle with and without shadowing compared to wind-tunnel data 


\section{IV.B. Space Shuttle}

A geometry mesh for the Space Shuttle, modified to be compatible with the method selection algorithm has been used to generate aerodynamic coefficients. The resulting coefficients were then compared to those obtained from wind tunnel data. ${ }^{23}$ Although these data are known to contain some discrepancies when compared to flight data, they comprise a comprehensive and consistent set of data that can be used to compare the coefficients generated here at more data points than could be done using only flight data. The reference quantities of the coefficients are:

$S_{\text {ref }}=249.441 \mathrm{~m}^{2}, c_{\text {ref }}=12.0579 \mathrm{~m}, \mathbf{r}_{\mathbf{c o g}}=(21.356,0,0.8224) \mathrm{m}$

with the Orbiter nose as origin, positive $x$-direction rearwards and positive $z$-direction upwards

The analysis is performed using the default method-selection criteria given in Section III.B. When comparing the lift and drag coefficients, it can be seen that the method described here predicts the correct coefficients quite well. In addition, for low Mach numbers where a non-Newtonian method is used as the compression method, the accuracy of the method selection yields results which are clearly superior to a modified Newtonian approach, showing that the approach taken here will be useful for the calculation of the translational motion of the Space Shuttle. The effect of shadowing on the longitudinal force coefficients appears to be minimal, however.

The moment coefficients differ more strongly from the database values, as was to be expected from information from literature. ${ }^{12}$ However, it would be advisable to perform a more in-depth analysis of the actual flight data from the Space Shuttle, since there is a known discrepancy in the wind tunnel data, giving a consistent underprediction in the moment coefficients at high Mach numbers. This effect has been studied in detail ${ }^{22}$ and a build-up of the actual moment coefficient is shown here in Fig. 8(b). Since the results produced in the present study show an over-prediction of the coefficients at $\alpha>20^{\circ}$, they may approximate the actual coefficients more closely than can be seen from the figures here. The effect of shadowing is more noticeable here, with questionable influence on the accuracy of the results.

The stability derivatives of the lateral coefficients for small sideslip angles, which are important in the analysis of the vehicle stability during re-entry can be seen in Figs. 9. Here, the effect of the shadowing can be seen very clearly, in the case of the side force and yaw moment, they cause the trend of the coefficients to be followed quite closely, as opposed to the case without shadowing, where no such trend can be observed. The offset between the wind tunnel data and the results that include shadowing can be explained by the fact that the hard distinction between shadowing and no shadowing will not be quite as abrubt in reality. The streamlines flow which passes over the vehicle's edges will deflect and influence the external flow. In addition, vortices will form at these positions, further influencing the flow field.

In the case of the roll-moment derivative shown in Fig. IV.A, the influence of the shadowing is not quite as clearly positive, causing the coefficients to be somewhat underpredicted instead of overpredcited in most cases. This will, however, make for a more conservative estimate of the vehicle stability.

For the control increments, proper prediction of the pitch-moment increments is the primary objective for this study, since these values determine the deflection of the control surfaces to trim the vehicle. The resulting lift and drag increments can constitute $\sim 10 \%$ of the total lift and drag and should not be neglected, though. The control derivatives shown in Figs. 10-12 indicate that the predictions for the elevon increments are reasonably good, with lift increments having the lowest accuracy, showing differences of up to about 0.01 .

The body flap increments shown in Figs. 13-15 show greater discrepancies, however, for positive deflections. This can be caused by a number of factors. Firstly, for low deflection angles, the body flap will be fully engulfed in the fuselage boundary layer, reducing the body flap effectiveness. For higher deflection angles, though, the compression shock which will occur on the ramp between the fuselage and shock wave, which will cause an increase in surface pressure on the body flap. This effect is not observed as strongly on the elevon, however, where a similar ramp effect is expected to occur. Due to the smaller size of the wings, however, the boundary layer will be less thick on the elevons, reducing its effect on the control increments. Since a positive body flap deflection is required for Space Shuttle trim, these errors in the moment increment prediction will result in an over-prediction of the required body flap deflection. The effect on the performance of the Shuttle will be discussed in the next section.

Concluding, it can be said that the longitudinal force coefficients show excellent agreement with windtunnel data. The pitch moment coefficients are reasonably well predicted, but show substantial discrepancies 

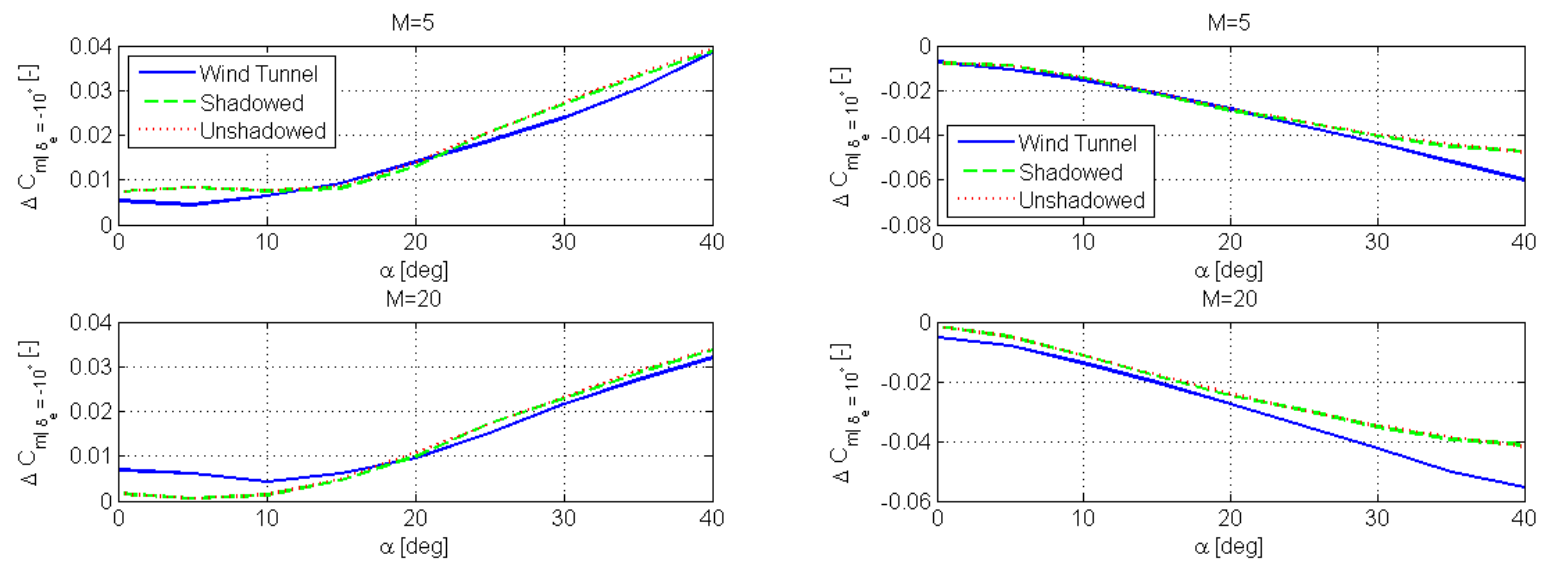

(a)

(b)

Figure 10. Elevon moment increments produced for the Space Shuttle compared to wind-tunnel data
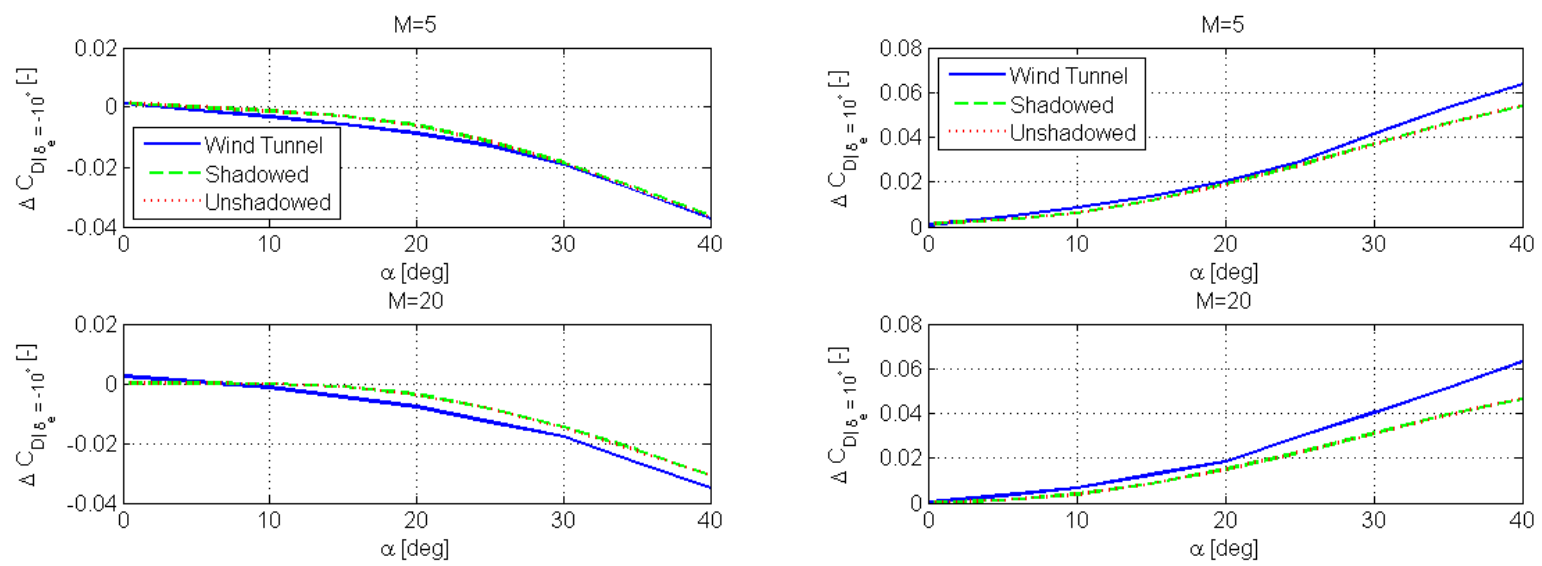

(a)

(b)

Figure 11. Elevon drag increments produced for the Space Shuttle compared to wind-tunnel data
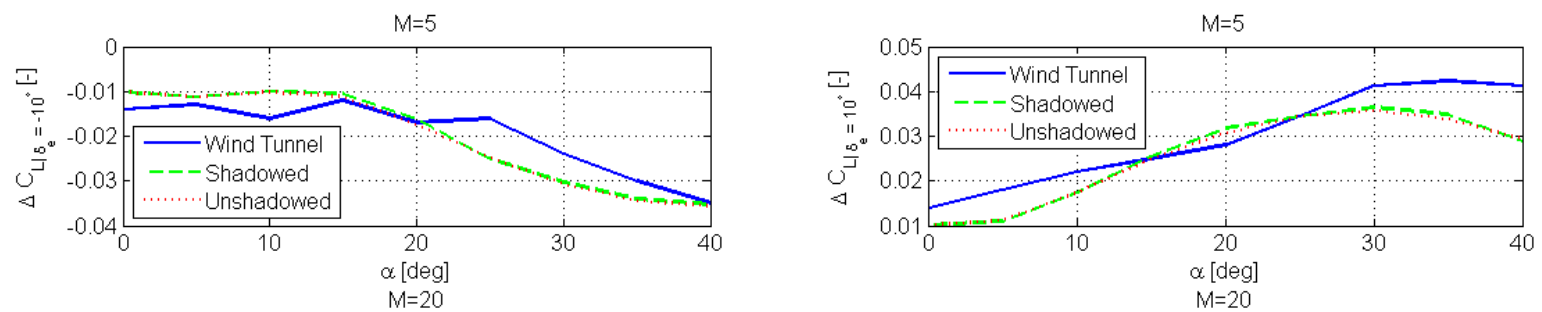

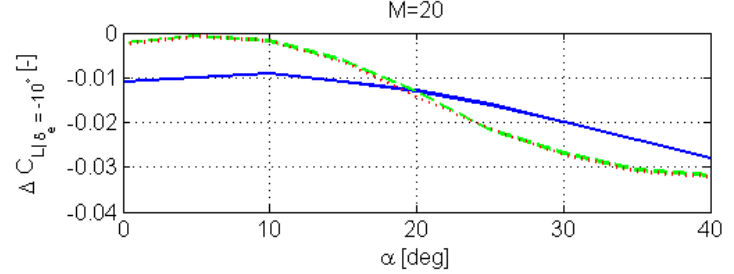

(a)

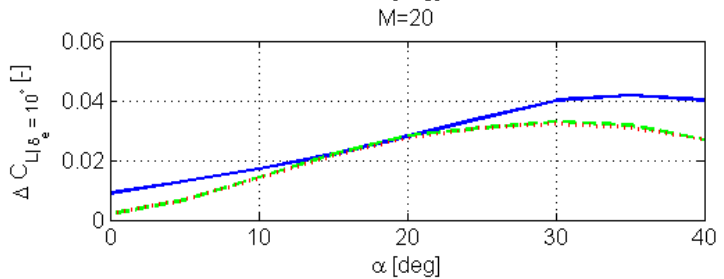

(b)

Figure 12. Elevon flap lift increments produced for the Space Shuttle compared to wind-tunnel data 

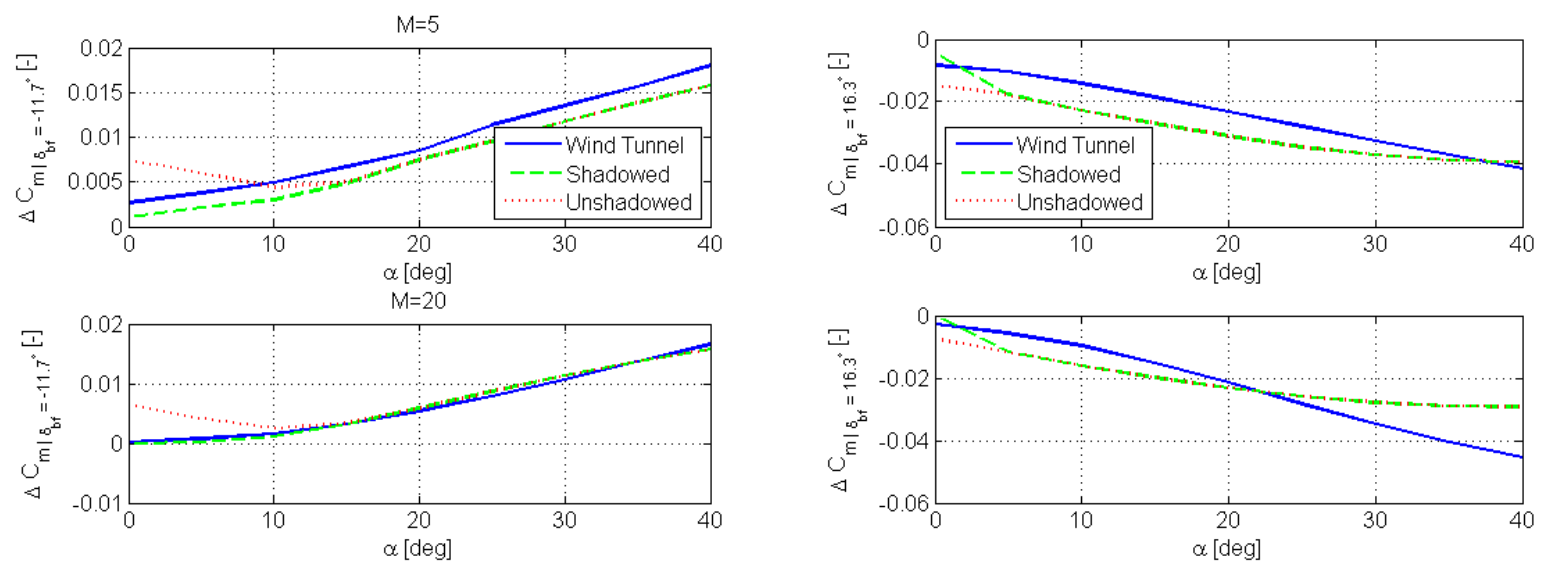

(a)

(b)

Figure 13. Body flap moment increments produced for the Space Shuttle compared to wind-tunnel data
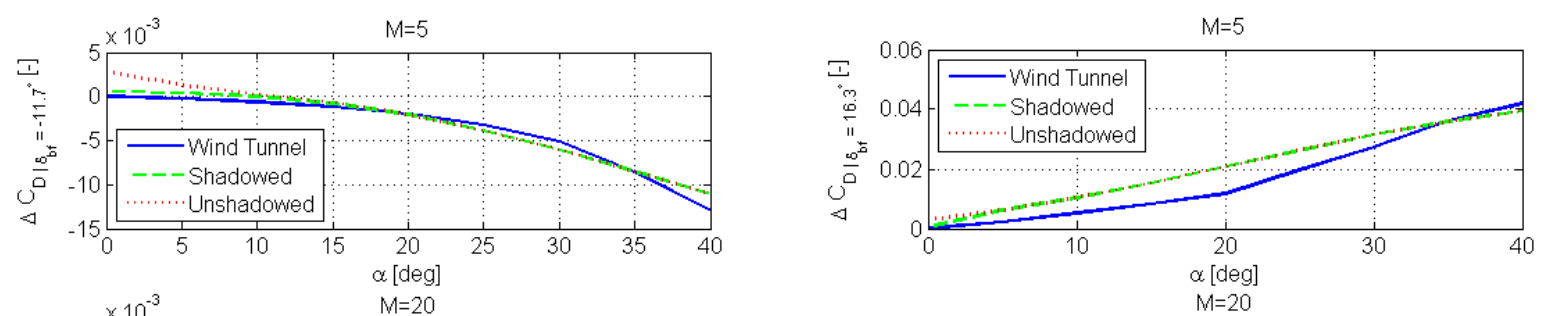

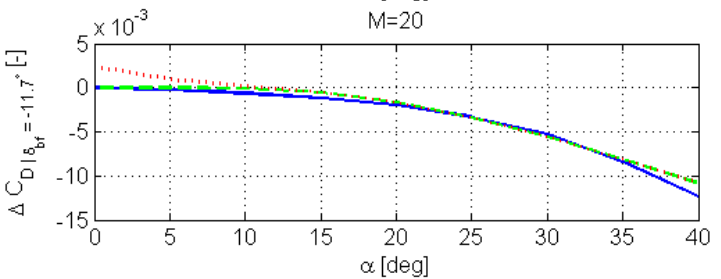

(a)

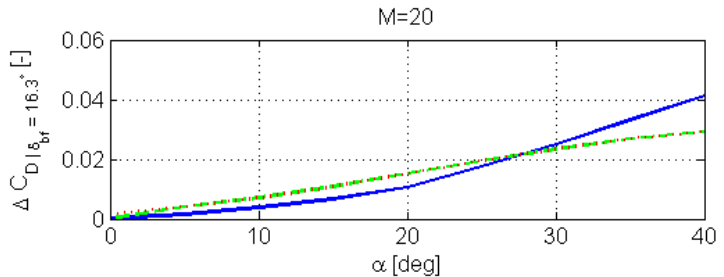

(b)

Figure 14. Body flap drag increments produced for the Space Shuttle compared to wind-tunnel data
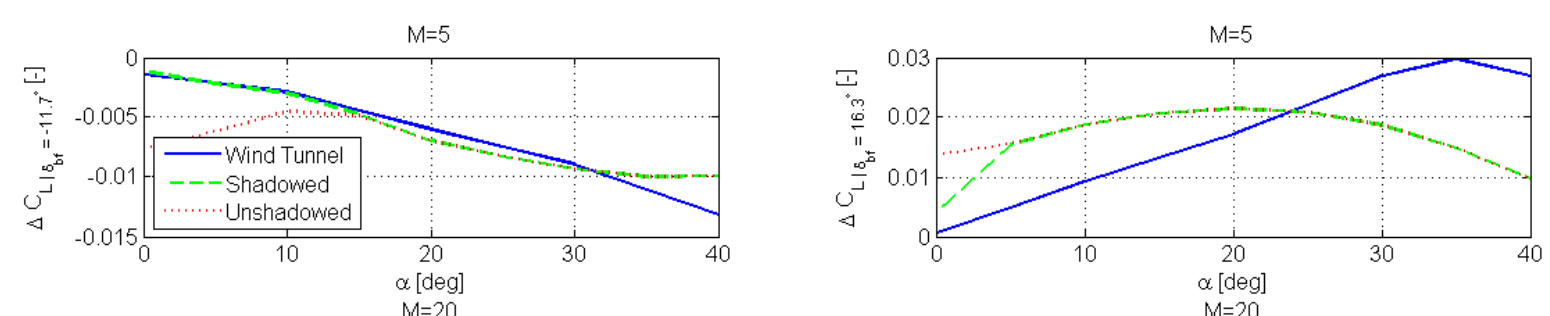

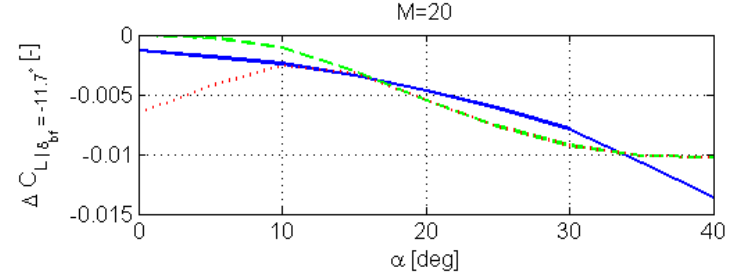

(a)

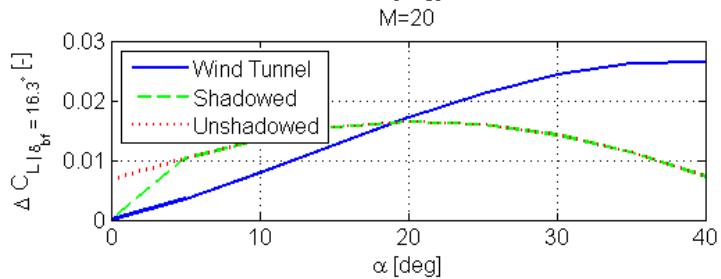

(b)

Figure 15. Body flap lift increments produced for the Space Shuttle compared to wind-tunnel data 
in some regions. In order to properly predict the side force and yaw moment coefficients, using shadowing is necessary, although this slightly decreases the accuracy of the yaw moment coefficients. Elevon increments are better predicted than body flap increments. The body flap moment and lift increments show the highest deviations, especially at high angles of attack and Mach numbers.

\section{Flight Mechanics}

In order to analyze the influence of the differences between the coefficients generated here and the wind tunnel data, 3DOF trajectories for an Apollo-shaped capsule and a Space-Shuttle shaped winged entry vehicle are generated using both databases. Section V.A will describe the assumptions on the re-entry environment and Section V.B will describe the guidance algorithms that are used for the entries.

\section{V.A. Environment Model}

The force models, which are included in the analysis, are limited to the aerodynamic force and a simplified gravity field model. This model includes the central gravity term, as well as the $J_{2}$ term. Since only unpowered entries are considered, no thrust force is required for the model. Other perturbative forces are neglected as their influence is limited in an entry trajectory, and the errors induced by their neglections are only small compared to errors due to inaccuracies in the aerodynamic coefficients. Integration of the equations of motion will be performed in inertial Earth-centered coordinates. The resultant equations of motion can be found in, for instance, ${ }^{24}$

For the determination of the atmospheric properties the 1976 Standard Atmosphere ${ }^{25}$ will be used, so that the atmospherics characteristics are a function of altitude only. No wind model is included, so that the atmosphere is assumed to rotate with the Earth.

The trajectory will be propagated until $M_{\infty}=3$ is reached, since below this Mach number, the aerodynamic coefficients which are calculated can no longer assumed to be valid.

For the defintion of the guidance algorithm discussed in the following section, simplified equations for the time derivative of the velocity and flight path angle are used. A spherical Earth with only a central gravity term is assumed. The centrifugal term due to the Earth's rotation is neglected, but the Coriolis term is included, as it has an appreciable influence in the hypersonic phase. Although its magnitude becomes lower than that of the centrifugal term for low velocities, such velocities will not be encountered for the trajectories generated here, since $M_{\min }=3$. The resulting equations become ${ }^{26}$ :

$$
\begin{aligned}
\frac{d V}{d t} & =-\frac{\rho S C_{D} V^{2}}{2 m}-g \sin \gamma \\
V \frac{d \gamma}{d t} & =\frac{\rho S C_{L} V^{2}}{2 m} \cos \sigma-\left(g-\frac{V^{2}}{r}\right) \cos \gamma+2 \omega_{P} V \cos \delta \sin \chi
\end{aligned}
$$

\section{V.B. Guidance and control approach}

To limit the computational time required for the trajectory calculation, targeting of a landing site or TAEM interface is not foreseen to be used in the present work. Instead, entry conditions will be specified, while the end conditions will be kept free. A trajectory optimization is deemed infeasible, since this would require an optimization for each of the vehicle shapes which is generated. Since the optimization of a single trajectory can be rather time-consuming, such an approach is unlikely to be cost-effective here.

The guidance and control approach differs between the ballistic and winged entry vehicles. For the ballistic vehicle, trimmed conditions will be assumed, so that:

$$
\alpha_{t r}=\left.\alpha\right|_{C_{m}=0}
$$

For symmetric vehicles with the center of gravity on the centerline, this will result in $\alpha=0$. For an offset center of gravity, as was the case for, for instance, the Apollo capsule, the trimmed angle of attack will be non-zero. Since the moment coefficient curve is a function of Mach number, the trimmed angle will be (lightly) dependent on Mach number. Since the trajectory propagation is 3 DOF, the time-dependent process by which the capsule changes attitude is not analyzed, but is assumed to be instantaneous. Considering the minor changes in angle of attack that are expected to occur, this is not expected to seriously influence the results, assuming vehicle stability. Attitude stability of the vehicle is important, however, as instability will 
make it unlikely for the vehicle to retain its trimmed conditions throughout the flight. For this reason, the following condition will be imposed on the capsule:

$$
\left.C_{m_{\alpha}}\right|_{\alpha=\alpha_{t r}}<0
$$

Whether this relation is fulfilled or not depends on the location of the center of gravity with respect to the center of pressure. Since the center of gravity is not known exactly from only the vehicle's shape, some variability in internal mass distribution could be used in order to produce a stable vehicle. A good criterion for static stability will be further investigated in future work. As dynamic derivatives are not determined in the analysis, dynamic stability will be assumed for all shapes. The bank angle of the capsule is determined by imposing $\dot{\gamma}<0$, which is derived from Eq. (13) as follows:

$$
\cos \sigma=\frac{m}{L}\left(g\left(1-\frac{V^{2}}{V_{c}^{2}}\right) \cos \gamma-2 \omega_{P} V \cos \delta \sin \chi\right)
$$

The winged vehicle shapes will be guided to fly a maximum time at a given reference stagnation point heat rate $q_{c, s, r e f}$, which can be seen as a typical mission profile for a class of experimental vehicles. Such an approach was found in literature ${ }^{27}$ and it, as well as a variant of it, are considered here. This approach has the virtue of allowing much of the guidance law to be expressed analytically. For the first portion of the trajectory, the vehicle is commanded to fly at maximum angle of attack to minimize the maximum stagnationpoint heat rate. After the heating peak, the heat-rate tracking is activated when $q_{c, s, r e f}$ is reached, which will guide the vehicle to maintain a constant heat rate. If the heat rate at the heating peak is smaller than $q_{c, s, r e f}$, the heat rate at the heating peak will be tracked. A similar approach could be used at the end of the trajectory to maintain constant dynamic pressure, as both rely (approximately) on keeping the following term:

$$
K=\rho V^{n}
$$

constant, where $n \approx 6$ (exact value depends on the choice of model, see Eq. (10)) for constant heat rate and $n=2$ for constant dynamic pressure. This leads to the following relation:

$$
\frac{d V}{d t}=-\frac{1}{n} \frac{V}{\rho} \frac{d \rho}{d t}
$$

Two approaches to enforce this are considered, with the latter derived as a variant of the former:

1. Modulate $\sigma$ to cause the drag to be such that Eq. (18) is enforced by using Eq. (13). Set $\alpha$ to value of $\left(\frac{L}{D}\right)_{\max }$ to maximize range. ${ }^{27}$

2. Modulate $\alpha$ to cause the drag to be such that Eq. (18) is enforced by using Eq. (12). The bank angle is modulated to enforce Eq. (16).

For the latter of these, a deviation in $\alpha$ at the initiation of the tracking of $K$ will cause deviations in the value that is to be tracked, whereas deviations in $\gamma$ will cause this in the case of the former of the guidance schemes. The mismatch of $\gamma$ at the initiation of the guidance algorithm has been found to cause much greater discrepancies than mismatches in $\alpha$ due to the fact that $\alpha$ can be changed more rapidly than $\gamma$, relative to the size of the respective errors, which are expected to occur. Although this error could be handled by using a PI controller to steer the vehicle to the proper heat-rate tracking, this would require automatic generation of the control gains for each of the randomly generated entry shapes in case of shape optimization. This makes the guidance algorithm less suitable to be included in the inner loop of such an optimization. For this reason, the value of $K$ is to be tracked by the former of the methods given.

To prevent the vehicle from skipping before it has reached the heat-rate peak, bank modulation to enforce Eq. (16) is used when the lift term becomes dominant in Eq. (13)

Neglecting the centrifugal term due to the rotation of the Earth and latitudinal dependency of the gravitational acceleration, this leads to the following relation for the drag:

$$
D_{K=\text { const }}=\frac{m V}{n \rho} \frac{d \rho}{d t}-m g \sin \gamma
$$

From the aerodynamic database that is created and the flight conditions, this value of the drag can be matched to a required angle of attack. A maximum angle of attack rate will be imposed to avoid discontinuities in the angle of attack. The winged vehicle shapes that are to be analyzed will have active pitch control 


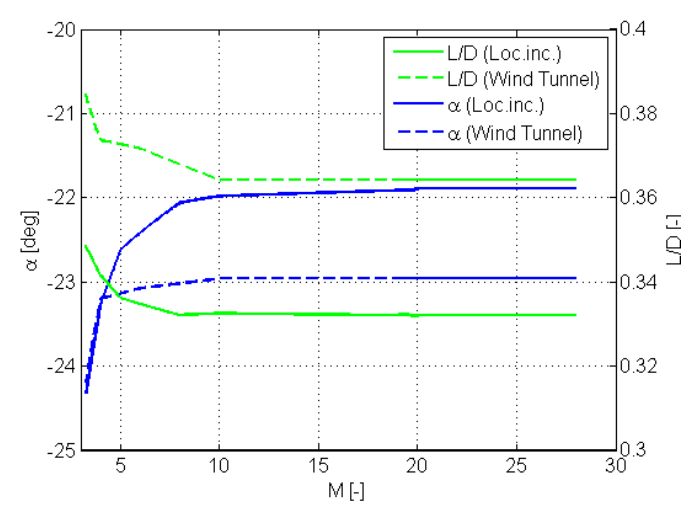

(a)

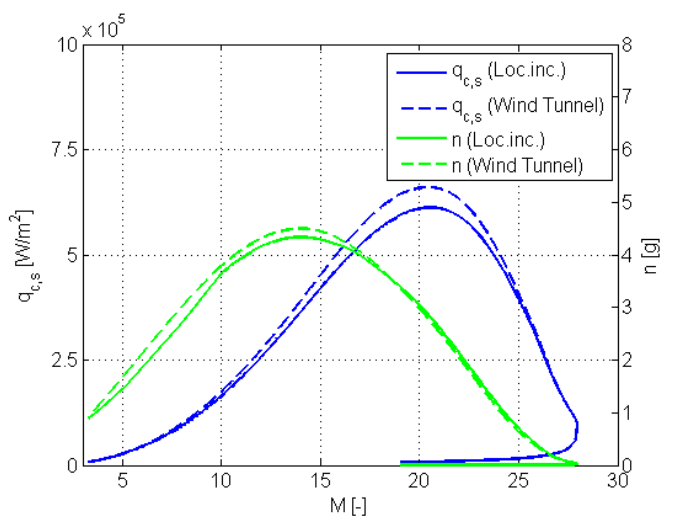

(c)

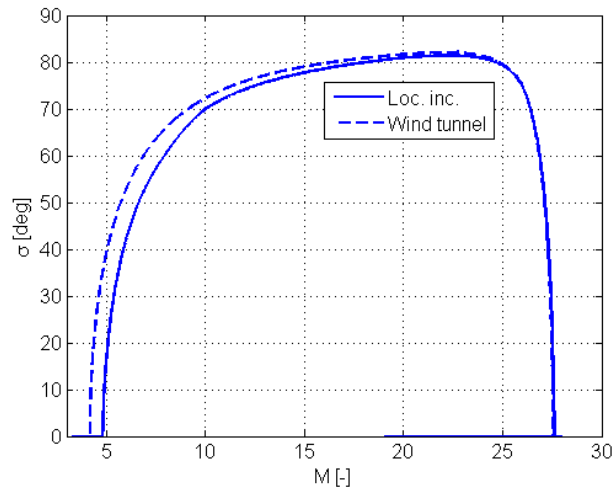

(b)

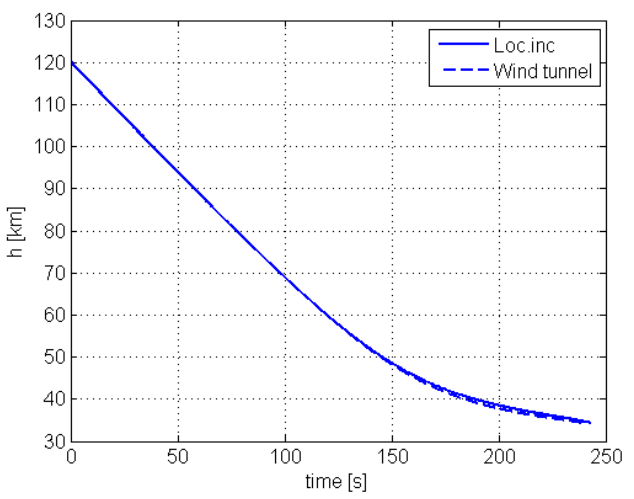

(d)

Figure 16. Re-entry trajectory profile of capsule shape a) Lift over drag and trimmed angle of attack, b) Bank angle c) Stagnation point heat rate and total g-load d) Altitude

capability by the use of a body flap and elevons. Since only symmetric $(\beta=0)$ entries are considered, the yaw and roll moments will be zero by virtue of the vehicle symmetry w.r.t. the vertical center plane. The guidance scheme will attempt to trim the pitch moment by body flap and elevon deflections. First, trim by only the body flap is attempted. If this fails, the elevons are also used.

\section{Trajectory results}

This section will describe the calculated entry trajectories of an Apollo-shaped capsule and a SpaceShuttle shaped lifting entry vehicles. The results obtained here are not meant to resemble the actual entry profiles of these two vehicles, but are instead used to assess the influence of errors in the aerodynamics on trajectory simulation, using assumptions and a guidance law similar to what can be used in a shapeoptimization process.

The trajectories were integrated using a Runge-Kutta $4^{\text {th }}$-order fixed step size integrator with a step size of $0.1 \mathrm{~s}$.

\section{VI.A. Capsule shape trajectory}

Fig. 16 shows the entry trajectory of the capsule, using the following initial conditions:

$h=120 \mathrm{~km}, \tau=225.5^{\circ}, \delta=-23.75^{\circ}, V_{R}=7.63 \mathrm{~km} / \mathrm{s}, \gamma=-4^{\circ}, \chi=49.6^{\circ}, m=4532 \mathrm{~kg}$.

It can be seen from Fig. 16(a) that the trimmed angle of attack remains approximately constant for the initial part of the re-entry, owing to the near Mach number independence of the modified Newtonian method at these Mach numbers. The gradual increase in the value of $\alpha$, and resultant decrease in $L / D$, is due to the 
Table 3. Comparison of results for performance criteria of Apollo to be used in optimization

\begin{tabular}{cccc}
\hline \hline & Wind tunnel & Local inclination & \% Percentage difference \\
\hline Stagnation point heat load & $49.232 \mathrm{MJ} / \mathrm{m}^{2}$ & $53.06 \mathrm{MJ} / \mathrm{m}^{2}$ & -7.2056 \\
Ground track length & $1425.6 \mathrm{~km}$ & $1430.2 \mathrm{~km}$ & +0.3242 \\
\hline
\end{tabular}

bridging between the low and high hypersonic regime for the aerodynamic coefficients. Additionally, for the final part of the entry, where the coefficients are calculated for low hypersonic speeds only, the Mach number independence is no longer present, since it no longer holds at these low velocities. This leads to the observed behavior that the trimmed angle of attack changes more substantially for $M<10$ than $M>10$. It should be noted, however, that the change in trimmed angle of attack is larger when using the local inclination methods than when using the wind-tunnel data. The difference in $L / D$ between the two cases is roughly constant $(\approx 0.03)$ over all angles of attack, though. This difference is likely to be acceptable for a conceptual design stage, as will be discussed shortly.

Bank-angle modulation can be seen to be required during part of the entry in Fig. 16(b). In the absence of this modulation, a small skip in the trajectory was observed, although not sufficiently large to exit the noticeable atmosphere. Additionally, the absence of limits on the attitude angles and angular rates can be seen to be justified, since there are no apparent discontinuities in the bank angle.

The figures show that the trajectories produced using the wind-tunnel coefficients and the ones generated here are very similar, as was to be expected from the discussion in the previous section. Since the $L / D$ is somewhat overpredicted, the vehicle's range and stagnation-point heat load (integrated heat flux) are also overpredicted. However, comparing these values, shown in Table 3, implies that the results are of sufficient accuracy to be useful in a conceptual design study. The error in the vehicle's range is very small, owing to the limited lifting capability of the vehicle. The error in stagnation point heat load, although substantially higher than for the range is still at an acceptaible low at about $7.5 \%$ for a conceptual study.

\section{VI.B. Shuttle shape trajectory}

Fig. 17 shows the trajectory of the Space Shuttle geometry using the guidance algorithm discussed in section V.B. The following initial conditions are used:

$h=120 \mathrm{~km}, \tau=225.5^{\circ}, \delta=-23.75^{\circ}, V_{R}=7.63 \mathrm{~km} / \mathrm{s}, \gamma=-1.5^{\circ}, \chi=49.6^{\circ}, m=75000 \mathrm{~kg}$

The following constraints are imposed on the attitude angles:

$10^{\circ}<\alpha<40^{\circ},|\dot{\alpha}|<2^{\circ} / s,|\sigma|<80^{\circ}$.

It can be clearly seen that the heat rate, which is set at a reference value of $500 \mathrm{~kW} / \mathrm{m}^{2}$, is properly tracked, even without the inclusion of control gains. This indicates that it is likely that the guidance scheme can be applied to a generic vehicle without the trajectory being sensitive to the gains. This makes the comparison between various entry shapes more fair, since the control gains will not positively or negatively influence the performance of different shapes.

In addition, it can be seen that the heat-rate tracking stops being possible once $\alpha$ reaches $10^{\circ}$, since at this point the drag required for constant heat rate becomes so low that it is impossible for the vehicle to attain. Also, it can be seen that the bank-angle modulation successfully prevents the vehicle from skipping out of the atmosphere before the heat-rate peak.

As can be seen from Fig. 17(b), the body flap is capable of trimming the Shuttle throughout most of the entry, as was to be expected. The correspondence to the actual deflections during the initial phase of the STS-1 entry are reasonable, as can be observed from Fig. 18. Due to the different control schemes used in actual flight and the simulations here, the correspondence only occurs at high Mach numbers, where the $\alpha$ profile is similar $\left(\approx\right.$ constant at $\left.40^{\circ}\right)$. Although it may appear curious that trim is achieved by a downward deflection of the body flap, which will cause a negative pitch moment increment, in light of Fig. 8, this is due to different centers of gravity during the wind-tunnel tests and re-entry. In the wind-tunnel tests, the center of gravity is approximately 24 inches more forward then during the entry of STS- $1 .{ }^{28}$ This change causes the center of pressure to lie in front of the center of gravity, instead of behind, as was the case during 


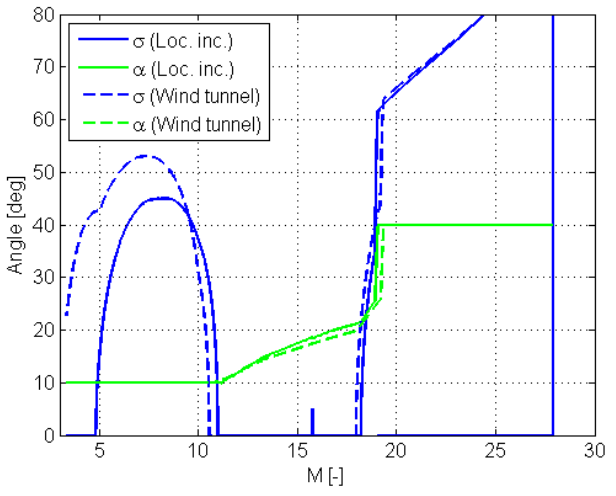

(a)

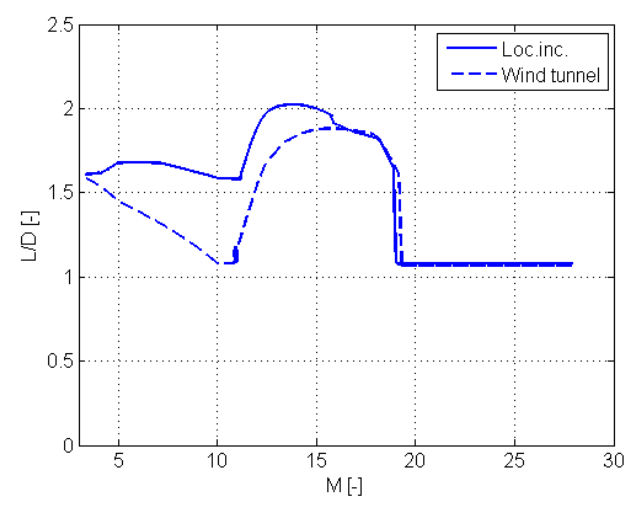

(c)

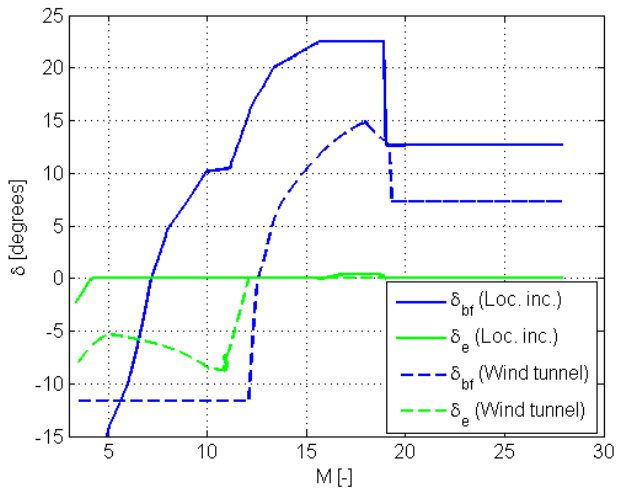

(b)

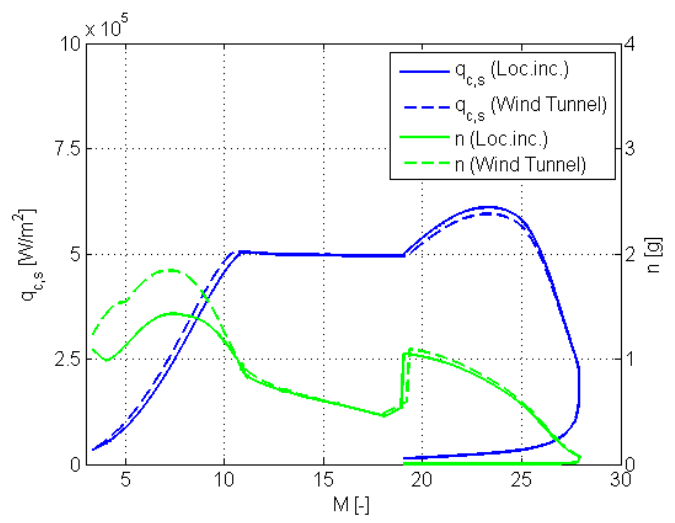

(d)

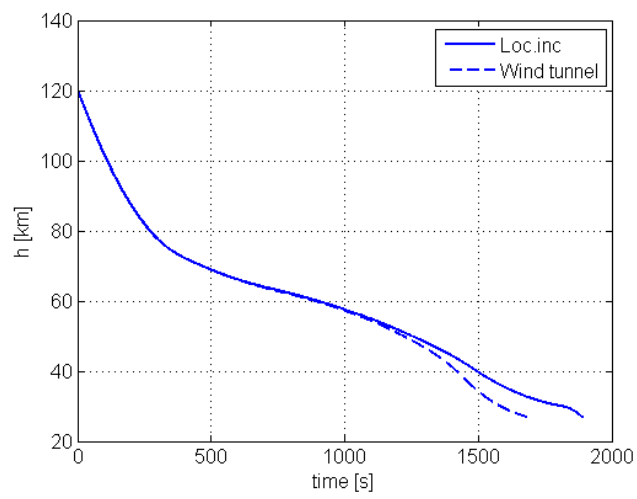

(e)

Figure 17. Space Shuttle re-entry trajectory profile a) Angles of attack and bank, b) Control surface deflections c) Lift over drag d) Stagnation point heat rate and total g-load e) Altitude 
Table 4. Comparison of results for performance criteria of the Space Shuttle to be used in optimization

\begin{tabular}{cccc}
\hline \hline & Wind tunnel & Local inclination & \% Percentage difference \\
\hline Stagnation point heat load & $698.9 \mathrm{MJ} / \mathrm{m}^{2}$ & $762.9 \mathrm{MJ} / \mathrm{m}^{2}$ & +9.160 \\
Ground track length & $9608 \mathrm{~km}$ & $10288 \mathrm{~km}$ & +7.0776 \\
\hline \hline
\end{tabular}

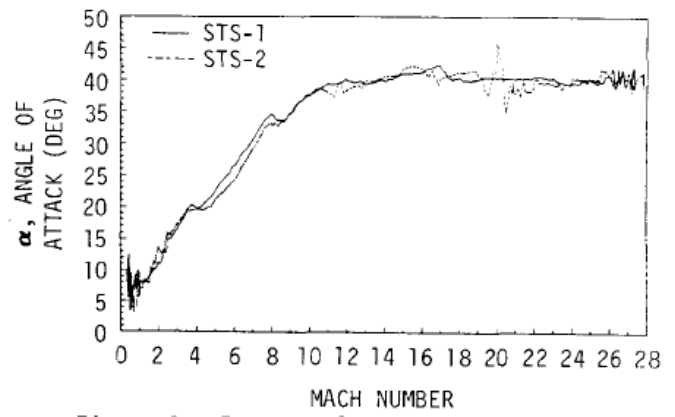

(a)

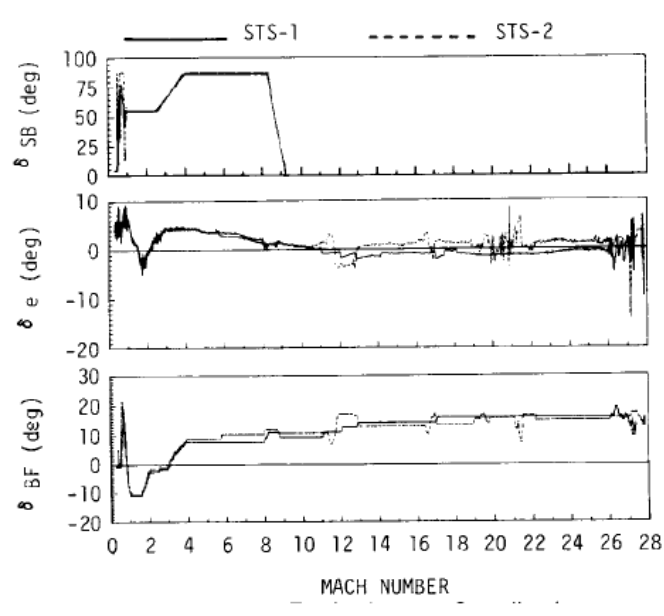

(b)

Figure 18. Selected re-entry conditions for STS-1 and STS-2 ${ }^{28}$ a) Angle-of-attack profile, b) Control-surface deflections

the wind tunnel tests, causing a positive untrimmed pitch moment.

The relatively large discrepancy in the behavior of the control-surface deflections for the two simulations is caused by the stacking of a number of errors. Namely, the over-prediction of the pitch moment at moderate angles of attack, along with the under-prediction of the body flap effectiveness, cause the required controlsurface deflections to show large differences during the nose-down maneuver. This is especially noticeable after the nose-down maneuver is initiated. Before this occurs, when $\alpha=40^{\circ}$ and the Mach number is high, the pitch moment difference is relatively small. This is also due to the fact that the angle-of-attack and bank-angle profiles have not begun to show different behavior yet in this region. After the initiation of the nose-down maneuver, though, the lower angle of attack causes the influence of the control surface deflections on $L / D$ to increase, in turn differently affecting the trajectories of the vehicle. This can be well seen in the $L / D$ curves of the two vehicles, which coincide well up until about Mach 16 , at which $\alpha \approx 20^{\circ}$.

The effect on the performance criteria is shown in Table 4. Despite the discrepacies in the $L / D$ curves, the difference in the ground track length is limited to about 7.5\%. The error in the stagnation-point heat load, although higher, is also an acceptably low at $10 \%$. This shows that despite the complexity of the Space-Shuttle shape and the resulting errors in the aerodynamic coefficients, vehicle performance is still predicted to a level of accuracy acceptable at an early design stage.

\section{Conclusions and Recommendations}

It has been shown that the aerodynamic models that have been implemented can predict the aerodynamic forces and moments for both ballistic and winged entry shapes sufficiently well for use in a conceptual design stage. The largest differences were observed for downward deflections of the Space-Shuttle body flap, due to the complex and highly inviscid flow, which will occur in this region. Prediction of the Space Shuttle moment coefficient was also less than satisfactory. This was also the case for the wind-tunnel data, however, as they showed a large discrepancy with flight data. A method of automatically analyzing a vehicle shape and selecting aerodynamic analysis methods is described, and is shown to select methods satisfactorily. The use of shielding of compression panels was shown to have limited influence on the body-force and -moment coefficients. The results for the body flap at low angles of attack, as well as the lateral stability derivatives, are strongly affected, though, with positive influence with the exception of the prediction of $C_{l_{\beta}}$. The trajectory analyses using simple guidance algorithms of a Space-Shuttle and Apollo shape were performed using both 
wind tunnel and results generated here, and both the ground-track length and the stagnation-point heat load differed by less than 10\%. This indicates the usefullness of the methods described in a conceptual design effort and shape optimization.

The methodology described here can be used for vehicle-shape optimization of either a capsule-shaped vehicle or a lifting entry vehicle. Both the aerothermodynamic characteristics and the vehicle trajectory will be generated using the methods described here.

\section{Appendix A: Method Selection Algorithm}

The method selection is performed per vehicle part and a single method per part is selected. If necessary, a part can be split into two parts on each of which a method is then selected. The criterion for determining whether a part is blunt or not is different for fusiform and planar parts (see Section II.B), since for a fusiform part it will typically involve the analysis of a nose and for a planar part of a leading edge. For both criteria, however, only the front most contour on the part is analyzed for bluntness determination. The average inclination $\bar{\theta}$ over a contour with $n$ panels is weighted by the panel areas, so:

$$
\bar{\theta}=\bar{A} \frac{1}{n} \sum_{i=0}^{n}\left(\frac{\theta_{i}}{A_{i}}\right)=\frac{1}{n^{2}}\left(\sum_{i=0}^{n} A_{i}\right)\left(\sum_{i=0}^{n}\left(\frac{\theta_{i}}{A_{i}}\right)\right)
$$

For a fusiform part, the most forward contour (denoted by a 0 subscript) must satisfy the following criterion:

$$
\bar{\theta}_{0}>k_{1}
$$

Values for $k_{1}$, as well as the other parameters on which the algorithm is based, are given in Table 5 .

For a planar part, the panels of the most forward contour are analyzed separately and a single panel is determined to be blunt if:

$$
\theta_{i}>k_{2}
$$

The contribution of a single panel to the bluntness of the entire contour is determined by its lateral extent. That is, its extent in longitudinal direction is neglected to scale the influence according to the number of streamlines that would impact the panel (in the Newtonian approximation). The relevant panel length $L_{p, i}$ for a panel becomes (see Fig. 19(a) ):

$$
L_{p, i}=\sqrt{\left(\frac{y_{i+1, j+1}+y_{i+1, j}}{2}-\frac{y_{i, j+1}+y_{i, j}}{2}\right)^{2}+\left(\frac{z_{i+1, j+1}+z_{i+1, j}}{2}-\frac{z_{i, j+1}+z_{i, j}}{2}\right)^{2}}
$$

The following summation over all blunt panels (according to Eq. (22)) is then performed to obtain the blunt fraction of the contour:

$$
n_{\text {blunt }}=\frac{\sum_{i: \theta_{i}>k_{2}} L_{i}}{\sum_{i=0}^{n} L_{i}}
$$

The part is considered blunt if:

$$
n_{\text {blunt }}>k_{3}
$$

Since the inviscid pressure far downstream of a blunt nose will be similar to that which is experienced if the geometry had a sharp nose (see previous section), these two cases will be treated similarly.

A blunt part is split if the downstream sections of the part have a sufficiently low inclination for a sufficiently large fraction to warrant the use of different methods on the two sections of the part. This criterion is quantified by finding the front-most lateral contour on which the following is satisfied:

$$
i_{\text {low }}=\min _{i}\left(\bar{\theta}_{i}<k_{4}\right)
$$

where, for a planar part, only panels on those longitudinal contours that were determined to have a blunt panel on lateral contour 0 are considered. In addition, for planar parts, the average is computed weighted by $L_{i}$. The part is not split immediately at this point, instead, it is checked whether the part of the vehicle to the rear of the vehicle is sufficiently large to warrant the split. In addition, non-blunt parts are analyzed by 


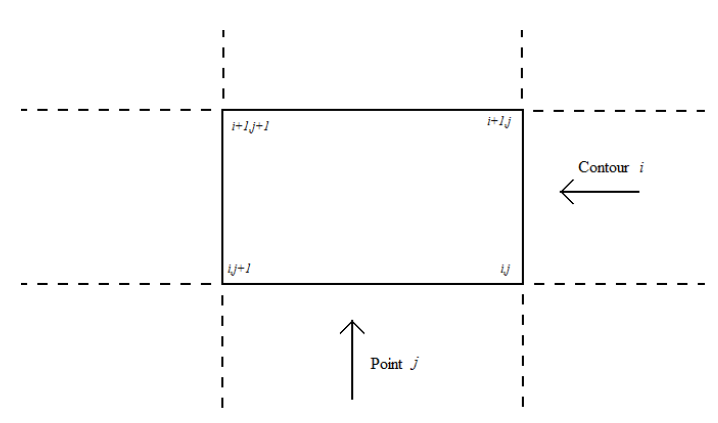

(a)

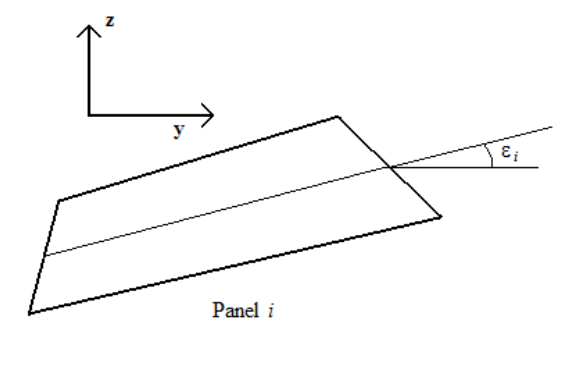

(b)

Figure 19. Schematic representation of a) Panel indices b) Lateral panel inclination angle $\epsilon$

Table 5. Choice of coefficients for method selection algorithm

\begin{tabular}{cc}
\hline \hline Parameter & Value \\
\hline$k_{1}$ & $45^{\circ}$ \\
$k_{2}$ & 30 \\
$k_{3}$ & $35^{\circ}$ \\
$k_{4}$ & $30^{\circ}$ \\
$k_{5}$ & 1.2 \\
$k_{6}$ & 40 \\
$k_{7}$ & $45^{\circ}$ \\
$k_{8}$ & 0.4 \\
$k_{9}$ & $45^{\circ}$ \\
\hline \hline
\end{tabular}

methods for which it is assumed that the shock wave is attached at the nose. As discussed in the previous section, being 'sufficiently far' downstream of the detached nose shock makes this approximation reasonable. For these reasons, the average $\mathrm{x}$-value of the contour where the part is to be split is determined as:

$$
\bar{x}_{\text {split }}=\bar{x}_{\text {low }}+k_{5}\left(\bar{x}_{\text {low }}-\bar{x}_{0}\right)
$$

Taking the buffer region size as a fraction of the blunt front of the part.

All of the above assumes a convex vehicle part, i.e. $\bar{\theta}_{i}$ is continuously decreasing with $i$. For, for instance, a Space Shuttle geometry, this assumption is invalidated by the presence of the front windows and engine nacelles. For this reason, a convexity check is included for the split point determination. If a convexity is found at contour for which:

$$
\bar{x}<k_{6} L
$$

where $L$ denotes the part length, a relevant convexity is identified. This criterion allows for including the front windows, but ignoring the nacelles in the split determination in the case of the Shuttle. If a convexity satisfying this criterion is found, the split is delayed until after this convexity.

Following the determination of blunt parts and any necessary splitting, the remaining non-blunt parts are further analyzed to determine whether to identify the part as 'flat' or 'curved'. A first check, which is considered here to be a sufficient, but not a necessary condition for 'flatness' of a single lateral contour, is the following (see Fig. 19(b)):

$$
\min _{i, j}\left|\epsilon_{i}-\epsilon_{j}\right|<k_{7}
$$

which indicates that the maximum change in lateral angle is sufficiently small over the whole part. This is not a necessary condition, however, as a wing or cube should also not be identified as round. To this end, a condition is included which analyzes the change in $\epsilon$ between each two consecutive panels and determines the lateral contour to be 'flat' if this change is sufficiently small on a sufficient number of panels on the lateral contour. The following derivative is used:

$$
\left(\frac{d \epsilon}{d L}\right) \approx \frac{\epsilon_{i+1}-\epsilon_{i}}{\frac{1}{2 \sum_{i=1}^{n} L_{i}} L_{i+1}-L_{i}}
$$


where the normalization of the denominator is performed to make the criterion independent of the total size of the lateral contour. The following is a criterion for 'flatness' over two panels:

$$
\left(\frac{d \epsilon}{d L}\right)_{i}<k_{8}
$$

The following is then used to determine the flatness of a lateral contour:

$$
\begin{aligned}
& n_{\text {flat }}=\frac{\sum_{\forall i:\left(\frac{d \epsilon}{d L}\right)_{i}<k_{2}} L_{i}}{\sum_{i=1}^{n} L_{i}} \\
& n_{\text {flat }}<k_{9}
\end{aligned}
$$

This last criterion is then used to determine the flatness of a lateral contour. An entire part is then considered blunt if this criterion, averaged over the entire part, is satisfied. It must be noted that the algorithm is still dependent on 9 parameters, for which an in-depth study of the sensitivity has not yet been performed. Table 5 gives a list of parameters which have been shown to perform well for a variety of geometries, however.

\section{References}

\footnotetext{
${ }^{1}$ Sudmeijer, K. and Mooij, E., "Shape Optimisation for a Small Experimental Re-entry Module," AIAA/AAAF 11th International Space Planes and Hypersonic Systems and Technology Conference, 2002.

${ }^{2}$ Farin, R., Curves and Surfaces for Computer Aided Geometric Design, A Practical Guide, Academic Press Inc., 3rd ed., 2003.

${ }^{3}$ Huertas, I., Mollmann, C., Weikert, S., and Martinez Barrio, A., "Re-entry Vehicle Design by Multidisciplinary optimisation in ASTOS," $4^{\text {th }}$ International Conference on Astrodynamics Tools and Techniques, 2010.

${ }^{4}$ Hirschel, E. and Weiland, C., Selected Aerothermodynamic Design Problems of Hypersonic Flight Vehicles, SpringerVerlag/AIAA, 2009.

${ }^{5}$ Craidon, C., "A Description of the Langley Wireframe Geometry Standard (LaWGS) Format, ," Tech. Rep. TM 85767, NASA, 1985.

${ }^{6}$ Ortega, G., Erb, S., Lopez, C., Laurel, C., Bueskens, C., Mooij, E., Laurel, F., Fliege, J., Giron-Sierra, J., Nunes Vicente, L., Burchell, M., Ruckmann, J., Gerdts, M., Lavagna, M., Soares Gil, P., and Martinez, R., "STA, the Space Trajectory Analysis Project," $4^{\text {th }}$ International Conference on Astrodynamics Tools and Techniques, 2010.

${ }^{7}$ Shaughnessy, J., Pinckney, S., and McMin, J., "Hypersonic Vehicle Simulation Model: Winged-Cone Configuration," Tech. Rep. TM-102610, NASA, 1990.

${ }^{8}$ Maughmer, M., Ozoroski, L., Ozoroski, T., and Straussfogel, D., "Prediction of Forces and Moments for Flight Vehicle Control Effectors - Final Report Part I: Validation for Predicting Hypersonic Vehicle Control Forces and Moments," Tech. Rep. CR-186571, NASA, 1990.

${ }^{9}$ Theisinger, J. and Braun, R., "Multi-Objective Hypersonic Entry Aeroshell Shape Optimization," Journal of Spacecraft and Rockets, Vol. 46, No. 5, 2009, pp. 957-966.

${ }^{10}$ Kinney, D., "Aero-Thermodynamics for Conceptual Design," $42^{\text {nd }}$ AIAA Aerospace Sciences Meeting and Exhibit, No. AIAA-2004-31, 2004.

${ }^{11}$ Cruz, C. and White, A., "Prediction of High-Speed Aerodynamic Characteristics Using the Aerodynamic Preliminary Analysis System," AIAA $7^{\text {th }}$ Applied Aerodynamics Conference, 1989.

${ }^{12}$ Anderson, J., Hypersonic and High-Temperature Gas Dynamics, AIAA Education Series, 2nd ed., 2006.

${ }^{13}$ Gentry, A., Smyth, D., and Oliver, W., The Mark IV Supersonic-Hypersonic Arbitrary Body Program, Volume II Program Formulation, 1973. 1981.

${ }^{14}$ Bonner, E., Clever, W., and Dunn, K., Aerodynamic preliminary analysis system 2. Part 1: Theory, NASA-CR-165627,

${ }^{15}$ Hoeijmakers, H., Sudmeijer, K., and Wegereef, J., "Hypersonic Aerothermodynamic Module for the NLRAERO Program," Tech. rep., Delft University of Technology, 1996.

${ }^{16}$ Maughmer, M., Ozoroski, L., Straussfogel, D., and Long, L., "Validation of Engineering Methods for Predicting Hypersonic Vehicle Control Forces and Moments," Journal of Guidance, Control and Dynamics, Vol. 16, No. 4, 1993, pp. 762-782.

${ }^{17}$ Moore, M. and Williams, J., "Aerodynamic Prediction Rationale for Analyses of Hypersonic Configurations," 27th Aerospace Sciences Meeting, 1989.

${ }^{18}$ Gomez, G., "Influence of Local Inclination Methods on the S/HABP Aerodynamic Analysis Tool," Tech. Rep. EWP 1793, esa, 1990.

${ }^{19}$ Tauber, M., Menees, G., and Adelman, H., "Aerothermodynamics of Transatmospheric vehicles," Journal of Aircraft, Vol. 24, No. 9, 1987, pp. 594-602.
}

${ }^{20}$ Anonymous, "Aerodynamic Data Manual for Project Apollo," Tech. Rep. NAS 9-159 (U), North American Aviation, 1965. 
${ }^{21}$ Massobrio, F., Viotto, R., Serpico, M., Sansone, A., Caporicci, M., and Muylaert, J.-M., "EXPERT: An atmospheric re-entry testbed," Acta Astronautica, Vol. 60, No. 12, 2007, pp. 974-985.

${ }^{22}$ Maus, J., Griffitj, B., and Szerna, K., "Hypersonic Mach Number and Real Gas Effects on Space Shuttle Orbiter Aerodynamics," Journal of Spacecraft and Rockets, Vol. 21, No. 2, 1983, pp. 136-141.

${ }^{23}$ Anonymous, "Aerodynamic Design Data Book, Volume1, Orbiter Vehicle, STS-1," Tech. Rep. SD72-SH-0060, Rockwell International, 1965.

${ }^{24}$ Vinh, N., Busemann, A., and Culp, R., Hypersonic and Planetary Entry Flight Mechanics, The University of Michigan Press, 1980.

${ }^{25}$ NOAA/NASA, "US Standard Atmosphere, 1976," Tech. rep., U.S. Government Printing Office, 1976.

${ }^{26}$ Mooij, E., Aerospace Plane Flight Dynamics: Analysis of Guidance and Control Concepts, Ph.D. thesis, TU Delft, 1998.

${ }^{27}$ Mooij, E. and Hänninen, P., "Distributed Global Trajectory Optimization of a Moderate Lift-to-Drag Re-entry Vehicle," AIAA Guidance, Navigation and Control Conference, No. AIAA 2009-5770, 2009.

${ }^{28}$ Underwood, J. and Cooke, D., "A Preliminary Correlation of the Orbiter Stability and Control Aerodynamics from the first two Space Shuttle Flights (STS-1 \& 2) with Preflight Predictions," 12 ${ }^{\text {th }}$ Aerodynamic Testing Conference, No. AIAA 82-0564, 1982. 